Louisiana State University

LSU Digital Commons

Faculty Publications

Department of Physics \& Astronomy

$1-1-2019$

\title{
KELT-22Ab: A Massive, Short-Period Hot Jupiter Transiting a Near- solar Twin
}

Jonathan Labadie-Bartz

University of Delaware

Joseph E. Rodriguez

Harvard-Smithsonian Center for Astrophysics

Keivan G. Stassun

Vanderbilt University

David R. Ciardi

California Institute of Technology

Kaloyan Penev

The University of Texas at Dallas

See next page for additional authors

Follow this and additional works at: https://digitalcommons.Isu.edu/physics_astronomy_pubs

\section{Recommended Citation}

Labadie-Bartz, J., Rodriguez, J., Stassun, K., Ciardi, D., Penev, K., Johnson, M., Gaudi, B., Colón, K., Bieryla, A., Latham, D., Pepper, J., Collins, K., Evans, P., Relles, H., Siverd, R., Bento, J., Yao, X., Stockdale, C., Tan, T., Zhou, G., Eastman, J., Albrow, M., Bayliss, D., Beatty, T., Berlind, P., Bozza, V., Calkins, M., Cohen, D., Curtis, I., Esquerdo, G., Feliz, D., Fulton, B., \& Gregorio, J. (2019). KELT-22Ab: A Massive, Short-Period Hot Jupiter Transiting a Near-solar Twin. Astrophysical Journal, Supplement Series, 240 (1) https://doi.org/10.3847/ 1538-4365/aaee7e

This Article is brought to you for free and open access by the Department of Physics \& Astronomy at LSU Digital Commons. It has been accepted for inclusion in Faculty Publications by an authorized administrator of LSU Digital Commons. For more information, please contact ir@lsu.edu. 


\section{Authors}

Jonathan Labadie-Bartz, Joseph E. Rodriguez, Keivan G. Stassun, David R. Ciardi, Kaloyan Penev, Marshall C. Johnson, B. Scott Gaudi, Knicole D. Colón, Allyson Bieryla, David W. Latham, Joshua Pepper, Karen A. Collins, Phil Evans, Howard Relles, Robert J. Siverd, Joao Bento, Xinyu Yao, Chris Stockdale, Thiam Guan Tan, George Zhou, Jason D. Eastman, Michael D. Albrow, Daniel Bayliss, Thomas G. Beatty, Perry Berlind, Valerio Bozza, Michael L. Calkins, David H. Cohen, Ivan A. Curtis, Gilbert A. Esquerdo, Dax Feliz, Benjamin J. Fulton, and Joao Gregorio 
Swarthmore College

Works

Physics \& Astronomy Faculty Works

Physics \& Astronomy

$1-1-2019$

\title{
KELT-22Ab: A Massive, Short-Period Hot Jupiter Transiting A Near- Solar Twin
}

\author{
J. Labadie-Bartz
}

J. E. Rodriguez

K. G. Stassun

D. R. Ciardi

K. Penev

Eollow this and additional works at: https://works.swarthmore.edu/fac-physics

Part of the Astrophysics and Astronomy Commons

Let us know how access to these works benefits you

\section{Recommended Citation}

J. Labadie-Bartz, J. E. Rodriguez, K. G. Stassun, D. R. Ciardi, K. Penev, M. C. Johnson, B. S. Gaudi, K. D. Colón, A. Bieryla, D. W. Latham, J. Pepper, K. A. Collins, P. Evans, H. Relles, R. J. Siverd, J. Bento, X. Yao, C. Stockdale, T.-G. Tan, G. Zhou, J. D. Eastman, M. D. Albrow, D. Bayliss, T. G. Beatty, P. Berlind, V. Bozza, M. L. Calkins, David H. Cohen, I. A. Curtis, G. A. Esquerdo, D. Feliz, B. J. Fulton, J. Gregorio, D. James, Eric L.N. Jensen, J. A. Johnson, S. A. Johnson, M. D. Joner, D. Kasper, J. F. Kielkopf, R. B. Kuhn, M. B. Lund, A. Malpas, M. Manner, N. McCrady, K. K. McLeod, T. E. Oberst, M. T. Penny, P. A. Reed, D. H. Sliski, D. C. Stephens, D. J. Stevens, S. Villanueva Jr., R. A. Wittenmyer, J. T. Wright, and R. Zambelli. (2019). "KELT-22Ab: A Massive, Short-Period Hot Jupiter Transiting A Near-Solar Twin". Astrophysical Journal. Volume 240, Issue 1. DOI: 10.3847/1538-4365/aaee7e

https://works.swarthmore.edu/fac-physics/367

This work is brought to you for free by Swarthmore College Libraries' Works. It has been accepted for inclusion in Physics \& Astronomy Faculty Works by an authorized administrator of Works. For more information, please contact myworks@swarthmore.edu. 


\section{Authors}

J. Labadie-Bartz, J. E. Rodriguez, K. G. Stassun, D. R. Ciardi, K. Penev, M. C. Johnson, B. S. Gaudi, K. D. Colón, A. Bieryla, D. W. Latham, J. Pepper, K. A. Collins, P. Evans, H. Relles, R. J. Siverd, J. Bento, X. Yao, C. Stockdale, T.-G. Tan, G. Zhou, J. D. Eastman, M. D. Albrow, D. Bayliss, T. G. Beatty, P. Berlind, V. Bozza, M. L. Calkins, David H. Cohen, I. A. Curtis, G. A. Esquerdo, D. Feliz, B. J. Fulton, J. Gregorio, D. James, Eric L.N. Jensen, J. A. Johnson, S. A. Johnson, M. D. Joner, D. Kasper, J. F. Kielkopf, R. B. Kuhn, M. B. Lund, A. Malpas, M. Manner, N. McCrady, K. K. McLeod, T. E. Oberst, M. T. Penny, P. A. Reed, D. H. Sliski, D. C. Stephens, D. J. Stevens, S. Villanueva Jr., R. A. Wittenmyer, J. T. Wright, and R. Zambelli 


\title{
KELT-22Ab: A Massive, Short-Period Hot Jupiter Transiting a Near-solar Twin
}

\author{
Jonathan Labadie-Bartz (10) ${ }^{1,2,3}$, Joseph E. Rodriguez ${ }^{4}$ (1), Keivan G. Stassun ${ }^{5,6}$ (1) , David R. Ciardi ${ }^{7}$, Kaloyan Penev ${ }^{8}$ (1),
}

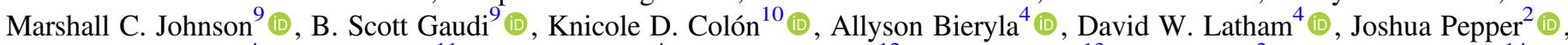
Karen A. Collins ${ }^{4}(0)$, Phil Evans ${ }^{11}$, Howard Relles ${ }^{4}$, Robert J. Siverd ${ }^{12}(1)$, Joao Bento ${ }^{13}$, Xinyu Yao ${ }^{2}(1)$, Chris Stockdale ${ }^{14}$, Thiam-Guan $\operatorname{Tan}^{15}$ (1) , George Zhou ${ }^{4,13}$ (1), Jason D. Eastman ${ }^{4}$ (1), Michael D. Albrow ${ }^{16}$ (1) , Daniel Bayliss ${ }^{13,17}$ (1), Thomas G. Beatty ${ }^{18,19}$ (10), Perry Berlind ${ }^{4}$, Valerio Bozza ${ }^{20,21}$ (1), Michael L. Calkins ${ }^{4}$ (무, David H. Cohen ${ }^{22}$, Ivan A. Curtis ${ }^{23}$, Gilbert A. Esquerdo ${ }^{4}$, Dax Feliz ${ }^{5,6}$ (1) , Benjamin J. Fulton ${ }^{24,42}$ (1) , Joao Gregorio ${ }^{25}$, David James ${ }^{26}$, Eric L. N. Jensen ${ }^{22}$ (1), John A. Johnson ${ }^{4}$, Samson A. Johnson ${ }^{9}$, Michael D. Joner ${ }^{27}$, David Kasper ${ }^{28}$, John F. Kielkopf ${ }^{29}$, Rudolf B. Kuhn ${ }^{30,31}$, Michael B. Lund ${ }^{5}$ (1), Amber Malpas ${ }^{16}$, Mark Manner ${ }^{32}$ (1), Nate McCrady ${ }^{33}$, Kim K. McLeod ${ }^{34}$ (1), Thomas E. Oberst ${ }^{35}$, Matthew T. Penny ${ }^{9}$ (D) Phillip A. Reed ${ }^{36}$ (1) , David H. Sliski ${ }^{37}$, Denise C. Stephens ${ }^{27}$, Daniel J. Stevens ${ }^{9}$ (i), Steven Villanueva, Jr. ${ }^{9}$ (i), Robert A. Wittenmyer ${ }^{38}$ (iD, J. T. Wright ${ }^{39,40}$ (i), and Roberto Zambelli ${ }^{41}$

${ }^{1}$ Department of Physics \& Astronomy, University of Delaware, Newark, DE 19716, USA; jbartz@udel.edu ${ }^{2}$ Department of Physics, Lehigh University, 16 Memorial Drive East, Bethlehem, PA 18015, USA

${ }^{3}$ Instituto de Astronomia, Geofísica e Ciências Atmosféricas, Universidade de São Paulo, Rua do Matão 1226, Cidade Universitária,05508-900, São Paulo, SP, Brazil

${ }^{4}$ Harvard-Smithsonian Center for Astrophysics, 60 Garden Street, Cambridge, MA 02138, USA

${ }^{5}$ Department of Physics and Astronomy, Vanderbilt University, Nashville, TN 37235, USA

${ }^{6}$ Department of Physics, Fisk University, 1000 17th Avenue North, Nashville, TN 37208, USA ${ }^{7}$ Caltech-IPAC/NExScI, Caltech, Pasadena, CA 91125, USA

${ }^{8}$ Department of Physics, The University of Texas at Dallas, 800 West Campbell Road, Richardson, TX 75080-3021, USA

${ }^{9}$ Department of Astronomy, The Ohio State University, 140 West 18th Avenue, Columbus, OH 43210, USA

${ }^{10}$ NASA Goddard Space Flight Center, Exoplanets and Stellar Astrophysics Laboratory (Code 667), Greenbelt, MD 20771, USA

${ }^{11}$ El Sauce Observatory, Chile

${ }^{12}$ Las Cumbres Observatory, 6740 Cortona Drive, Suite 102, Santa Barbara, CA 93117, USA

${ }^{13}$ Research School of Astronomy and Astrophysics, Mount Stromlo Observatory, Australian National University, Cotter Road, Weston, ACT 2611, Australia ${ }_{15}^{4}$ Hazelwood Observatory, Churchill, Victoria, Australia

${ }^{15}$ Perth Exoplanet Survey Telescope, Perth, Australia

${ }^{16}$ Department of Physics and Astronomy, University of Canterbury, Private Bag 4800, Christchurch, New Zealand

${ }^{17}$ Department of Physics, University of Warwick, Gibbet Hill Road, Coventry, CV4 7AL, UK

${ }_{18}$ Department of Astronomy \& Astrophysics, The Pennsylvania State University, 525 Davey Lab, University Park, PA 16802, USA

${ }^{19}$ Center for Exoplanets and Habitable Worlds, The Pennsylvania State University, 525 Davey Lab, University Park, PA 16802, USA

${ }^{20}$ Dipartimento di Fisica "E.R.Caianiello", Università di Salerno, Via Giovanni Paolo II 132, Fisciano I-84084, Italy

${ }^{21}$ Istituto Nazionale di Fisica Nucleare, Napoli, Italy

${ }^{22}$ Department of Physics \& Astronomy, Swarthmore College, Swarthmore, PA 19081, USA

${ }^{23}$ Ivan Curtis Private Observatory, Adelaide, Australia

${ }^{24}$ Division of Geological and Planetary Sciences, California Institute of Technology, Pasadena, CA 91101, USA

${ }^{25}$ Atalaia Group \& CROW Observatory, Portalegre, Portugal

${ }^{26}$ Event Horizon Telescope, Harvard-Smithsonian Center for Astrophysics, MS-42, 60 Garden Street, Cambridge, MA 02138, USA

${ }^{27}$ Department of Physics and Astronomy, Brigham Young University, Provo, UT 84602, USA

${ }^{28}$ Department of Physics \& Astronomy, University of Wyoming, 1000 E University Avenue, Dept 3905, Laramie, WY 82071, USA

${ }^{29}$ Department of Physics and Astronomy, University of Louisville, Louisville, KY 40292, USA

${ }^{30}$ South African Astronomical Observatory, P.O. Box 9, Observatory, 7935, Cape Town, South Africa

${ }^{31}$ Southern African Large Telescope, P.O. Box 9, Observatory, 7935, Cape Town, South Africa

${ }^{32}$ Spot Observatory, Nashville, TN 37206, USA

${ }^{33}$ Department of Physics and Astronomy, University of Montana, Missoula, MT 59812, USA

${ }^{34}$ Department of Astronomy, Wellesley College, Wellesley, MA 02481, USA

${ }^{35}$ Department of Physics, Westminster College, New Wilmington, PA 16172, USA

${ }^{36}$ Department of Physical Sciences, Kutztown University, Kutztown, PA 19530, USA

${ }^{37}$ Department of Physics and Astronomy, University of Pennsylvania, Philadelphia, PA 19104, USA

${ }^{38}$ University of Southern Queensland, Centre for Astrophysics, Toowoomba, QLD 4350 Australia

${ }^{39}$ Department of Astronomy \& Astrophysics, 525 Davey Laboratory, The Pennsylvania State University, University Park, PA, 16802, USA

${ }^{40}$ Center for Exoplanets and Habitable Worlds, 525 Davey Laboratory, The Pennsylvania State University, University Park, PA, 16802, USA

${ }^{41}$ Società Astronomica Lunae, Italy

Received 2018 March 19; revised 2018 October 11; accepted 2018 October 16; published 2019 January 21

\begin{abstract}
We present the discovery of KELT-22Ab, a hot Jupiter from the KELT-South survey. KELT-22Ab transits the moderately bright $(V \sim 11.1)$ Sun-like G2V star TYC 7518-468-1. The planet has an orbital period of $P=$ $1.3866529 \pm 0.0000027$ days, a radius of $R_{P}=1.285_{-0.071}^{+0.12} R_{J}$, and a relatively large mass of $M_{P}=3.47_{-0.14}^{+0.15} M_{J}$. The star has $R_{\star}=1.099_{-0.046}^{+0.079} R_{\odot}, M_{\star}=1.092_{-0.041}^{+0.045} M_{\odot}, T_{\text {eff }}=5767_{-49}^{+50} \mathrm{~K}, \log g_{\star}=4.393_{-0.060}^{+0.039}(\mathrm{cgs})$, and $[\mathrm{m} / \mathrm{H}]=+0.259_{-0.083}^{+0.085}$; thus other than its slightly super-solar metallicity, it appears to be a near-solar twin. Surprisingly, KELT-22A exhibits kinematics and a Galactic orbit that are somewhat atypical for thin-disk stars. Nevertheless, the star is rotating rapidly for its estimated age, and shows evidence of chromospheric activity. Imaging reveals a slightly fainter companion to KELT-22A that is likely bound, with a projected separation of
\end{abstract}


$6^{\prime \prime}$ ( $\sim 1400$ au). In addition to the orbital motion caused by the transiting planet, we detect a possible linear trend in the radial velocity of KELT-22A, suggesting the presence of another relatively nearby body that is perhaps non-stellar. KELT-22Ab is highly irradiated (as a consequence of the small semimajor axis of $a / R_{\star}=4.97$ ), and is mildly inflated. At such small separations, tidal forces become significant. The configuration of this system is optimal for measuring the rate of tidal dissipation within the host star. Our models predict that, due to tidal forces, the semimajor axis is decreasing rapidly, and KELT-22Ab is predicted to spiral into the star within the next Gyr.

Key words: methods: observational - planets and satellites: detection - planets and satellites: gaseous planets techniques: photometric - techniques: radial velocities - techniques: spectroscopic

Supporting material: data behind figures

\section{Introduction}

A large and rapidly increasing number of transiting exoplanets have been discovered in the recent years. The remarkable growth of this field was initially propelled by photometric ground-based surveys, such as HATNet (Bakos et al. 2004), SuperWASP (Pollacco et al. 2006), XO (McCullough et al. 2005), TrES (Alonso et al. 2004), MEarth (Nutzman et al. 2009; Berta et al. 2012), TRAPPIST (Gillon et al. 2017), and the Qatar Exoplanet Survey (Alsubai et al. 2013). Space-based missions, including CoRoT (Baglin et al. 2006), Kepler (Borucki et al. 2010), and K2 (Howell et al. 2014) have been exceptionally successful, discovering a wealth of planets and revealing that compact systems including small planets are common. The exoplanets that have been discovered are incredibly diverse, with many planets being unlike anything seen in the solar system. Such a wide range of planetary properties and system architectures provides an interesting challenge to theories that attempt to describe how planetary systems form, and how they have evolved into the configurations that we see today.

The parameter space of exoplanet systems is becoming increasingly populated as new discoveries are made. Groundbased transit surveys are well suited to identifying large, shortperiod gas-giant planets. These so-called "hot Jupiters" typically have masses $\gtrsim 0.25 M_{\mathrm{J}}$, radii between $\sim 1-2 R_{\mathrm{J}}$, and orbital periods $\lesssim 10$ days. Statistical studies of planetary populations have revealed that this class of planet is inherently rare, with only about $1 \%$ of Sun-like stars estimated to host a hot Jupiter (Wright et al. 2012).

The existence of hot Jupiters provides a unique challenge and a valuable diagnostic for theories that describe how planetary systems form and evolve. Broadly speaking, hot Jupiters were either formed at or near their current orbital configuration, or were formed farther out and then migrated inward (Dawson \& Johnson 2018). The processes by which a planetary system is formed and the physics that dictate its further evolution are becoming increasingly constrained as knowledge of planetary parameters improves and as better population statistics are derived from observational data. It is therefore important to discover and study exoplanets across a wide range of parameter space.

Understanding the properties of gas giants is an integral part of exoplanet science, particularly since they often dominate the mass and angular momentum budget of a planetary system. Although intrinsically rare, hot Jupiters are ideal targets for detailed characterization studies, especially when their host star is bright. The radius and mass of hot Jupiters can be measured with relatively high precision, revealing their bulk density. The atmospheric composition of these planets can be studied through transmission spectroscopy, where stellar light that passes through the planetary atmosphere during a transit is studied, revealing information about the atmosphere. Measuring orbital phase curves and secondary eclipses of hot Jupiters provides information about the albedo and global weather patterns for these highly irradiated planets. The physics of tidal interactions between host star and planet are amenable to study with close-in, massive planets. In addition to the scientific value of characterizing hot Jupiters, these endeavors serve the future of the field, as techniques are refined and technical challenges are addressed that push toward the ability to characterize smaller and more temperate worlds.

The Kilodegree Extremely Little Telescope (KELT) survey comprises two similar telescopes. KELT-North (Pepper et al. 2007) is located at Winer Observatory in Sonoita, Arizona, and KELT-South (Pepper et al. 2012) is situated at the South African Astronomical Observatory in Sutherland, South Africa. Both telescopes have a $42 \mathrm{~mm}$ aperture, a $26^{\circ} \times 26^{\circ}$ field of view, and a pixel scale of $23^{\prime \prime}$. The KELT survey is designed to detect giant transiting exoplanets, with optimal precision $(\lesssim 1 \%)$ for stars between $8 \lesssim V \lesssim 11$. The planetary systems discovered with KELT are particularly well suited for detailed characterization studies.

Here, we present the discovery of KELT-22Ab, a massive hot Jupiter on a short $P=1.39$ day orbit, transiting a Sun-like star, but metal-rich and with unusual kinematics, with a spectral type of $\mathrm{G} 2$ and a brightness of $V=11.1 \mathrm{mag}$.

In Section 2 we describe the survey data used to discover, and the follow-up observations used to confirm, KELT-22Ab. Section 3 presents properties of the host star. We use information from the literature and spectroscopically derived stellar parameters, along with various models, to put the system into context. The results of our global fit are shown in Section 4, and our false-positive analysis is explained in Section 5. We further discuss interesting aspects of the KELT22 system in Section 6, and conclude with Section 7.

\section{Discovery and Follow-up Observations}

\subsection{KELT-South Observations and Photometry}

The star TYC 7518-468-1 (hereafter KELT-22A) was observed by the KELT-South telescope, in KELT-South Field 32 (KS32), the J2000 central coordinates of which are $\alpha=0^{\mathrm{h}}$ $4^{\mathrm{m}} 4^{\mathrm{s}} .8 \delta=-29^{\circ} 49^{\prime} 58^{\prime \prime} 8$. This field was also the original commissioning field of the KELT-South telescope, which was later included as a field in normal telescope operation. Commissioning data were taken between UT 2009 September 16 and UT 2009 December 30, where the field was observed 2294 times. During normal science operation between UT 2011 August 24 and UT 2015 December 28, 2918 images were taken, for a total of 5212 observations (commissioning + normal science operation). After our standard data reduction 


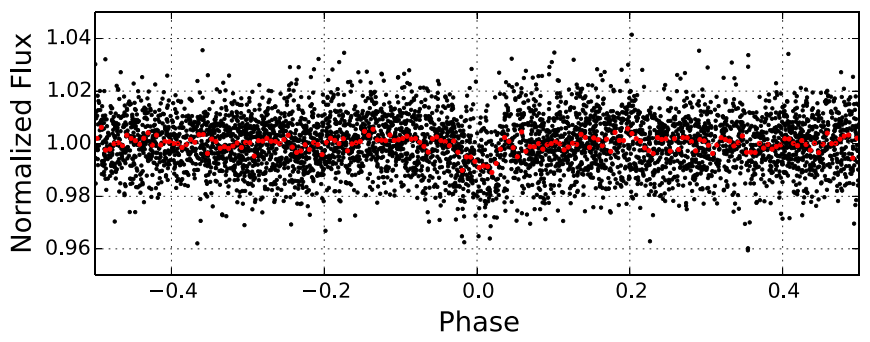

Figure 1. Discovery light curve for KELT-22A using 5049 observations from the KELT-South telescope phase-folded on the discovery period of 1.3866536 days. The red points are the data binned on a 5 minute timescale. The data used to create this figure are available.

routine, which includes removal of outliers and systematic and long-term trends, 5049 observations remain. These were analyzed, revealing a candidate transit signal with a period of 1.3866536 days and a depth of $\sim 0.7 \%$. The procedures for our data reduction and analysis, and candidate selection, are described in Kuhn et al. (2016). The discovery light curve, phased to the recovered period, is shown in Figure 1.

\subsection{Photometric Time-series Follow-up}

After identifying the candidate transit signal in the photometry from the KELT-South telescope, we then obtained follow-up observations with the KELT Follow-Up Network (KELT-FUN; Collins et al. 2018). These relatively highcadence, high-precision observations with larger telescopes allow us to reject various false-positive scenarios (e.g., a blended eclipsing binary) and serve to more precisely measure the depth, shape, and ephemeris of the transit event. The Tapir software package (Jensen 2013) was used to schedule the follow-up observations. The images were then reduced with the AstroImageJ (AIJ) software package ${ }^{43}$ (Collins \& Kielkopf 2013; Collins et al. 2017). A total of 11 transits (5 full and 6 partial) were observed between 2015 August and 2017 December. However, only the four highest-quality observations were included in our global fit, and are shown in Figure 2. The remaining seven light curves were consistent with a transit at the predicted ephemeris, but were not included in the global fit due to various issues (namely systematic effects and high photometric scatter).

KELT-22A has a close stellar companion, CCDM J233673437B (hereafter referred to as KELT-22B), that lies 6!" 1 to the southeast, and is about $0.45 \mathrm{mag}$ fainter in the $J$ band. These two sources are completely blended in the aperture used by KELT. After ruling out nearby eclipsing binaries (EBs) as the cause of the signal, an important aspect of our follow-up observations is to determine which of these two sources is actually being eclipsed. This was done by carefully placing small apertures around KELT-22A and KELT-22B separately in our follow-up photometry, and then extracting a light curve for each source. In each reduction of data done in this manner, an event is seen in KELT-22A, and no variability is seen in KELT-22B. Our analysis of radial velocity data corroborates this conclusion. However, light curves extracted this way are noisy, and are of insufficient quality for detailed analysis. We proceed by placing an aperture around both sources, extracting

\footnotetext{
${ }^{43}$ http://www.astro.louisville.edu/software/astroimagej/
}

a light curve for their combined flux, and finally correcting for the flux contamination of the companion.

With this approach, it is necessary to properly account for the flux from KELT-22B when determining the parameters of the KELT-22A system (e.g., Ciardi et al. 2015; Siverd et al. 2018). This "deblending" procedure is described in more detail in Section 4. The light curves shown in Figure 2 have been corrected in this fashion. Prior to the deblending process, transit depths are about a factor of 1.5 smaller.

In the following sub-sections, we describe the facilities that observed a transit of KELT-22A that resulted in a light curve of sufficient quality to incorporate into our global fit. We note that additional light curves from Hazelwood Observatory, PEST Observatory, and the MINERVA telescope array assisted in our confirmation of this as a genuine exoplanet system.

\subsubsection{Mt. John Observatory}

Mt. John Observatory is affiliated with the University of Canterbury, and is located in Lake Tekapo, New Zealand. The observatory employs a $0.61 \mathrm{~m}$ telescope with a $14^{\prime} \times 14^{\prime}$ field of view. KELT-22A was observed at Mt. John Observatory on UT 2015 August 26 using a Cousins V filter. This observation covers from approximately the onset of ingress to two hours past egress.

\subsubsection{El Sauce Observatory}

El Sauce Observatory is located near La Serena, Chile. The $0.356 \mathrm{~m}$ telescope has an 18 !.5 $\times 12$ !. 3 field of view. A full transit was covered on UT 2017 October 22 in a Cousins R filter, with about one hour each of pre-ingress and post-egress baseline.

\subsubsection{Las Cumbres Observatory (LCO)}

The transit on UT 2017 December 18 was observed with two of the LCO telescopes concurrently, one in SDSS i' and the other in Cousins I. Two $1 \mathrm{~m}$ telescopes located at the Cerro Tololo Inter-American Observatory (CTIO) in Cerro Tololo, Chile were used. The $1 \mathrm{~m}$ LCO telescopes at CTIO have a 4 $\mathrm{K} \times 4 \mathrm{~K}$ Sinistro detector with a $26^{\prime} \times 26^{\prime}$ field of view and a pixel scale of 0 " 389 per pixel.

\subsection{Spectroscopic Follow-up}

Spectroscopic observations of KELT-22A were obtained in order to measure the stellar parameters and its RV orbit. The relatively larger telescope apertures used for our spectroscopic observations resolve KELT-22A and KELT-22B, so the spectra are not contaminated by light from the neighboring source.

\subsubsection{Australia National University (ANU) $2.3 \mathrm{~m}$}

To approximately measure the stellar parameters of KELT$22 \mathrm{~A}$ and to place initial constraints on the dynamical mass of the orbiting body, we obtained reconnaissance spectroscopy with the WiFeS spectrograph mounted on the $2.3 \mathrm{~m}$ ANU telescope at Siding Spring Observatory (Dopita et al. 2007). $\mathrm{WiFeS}$ is an optical dual-beam, image-slicing integral fieldspectrograph. The design of the instrument allows us to extract a spectrum for both KELT-22A and KELT-22B from a single exposure. More information about the observing strategy and data reduction procedure is outlined in Bayliss et al. (2013). 


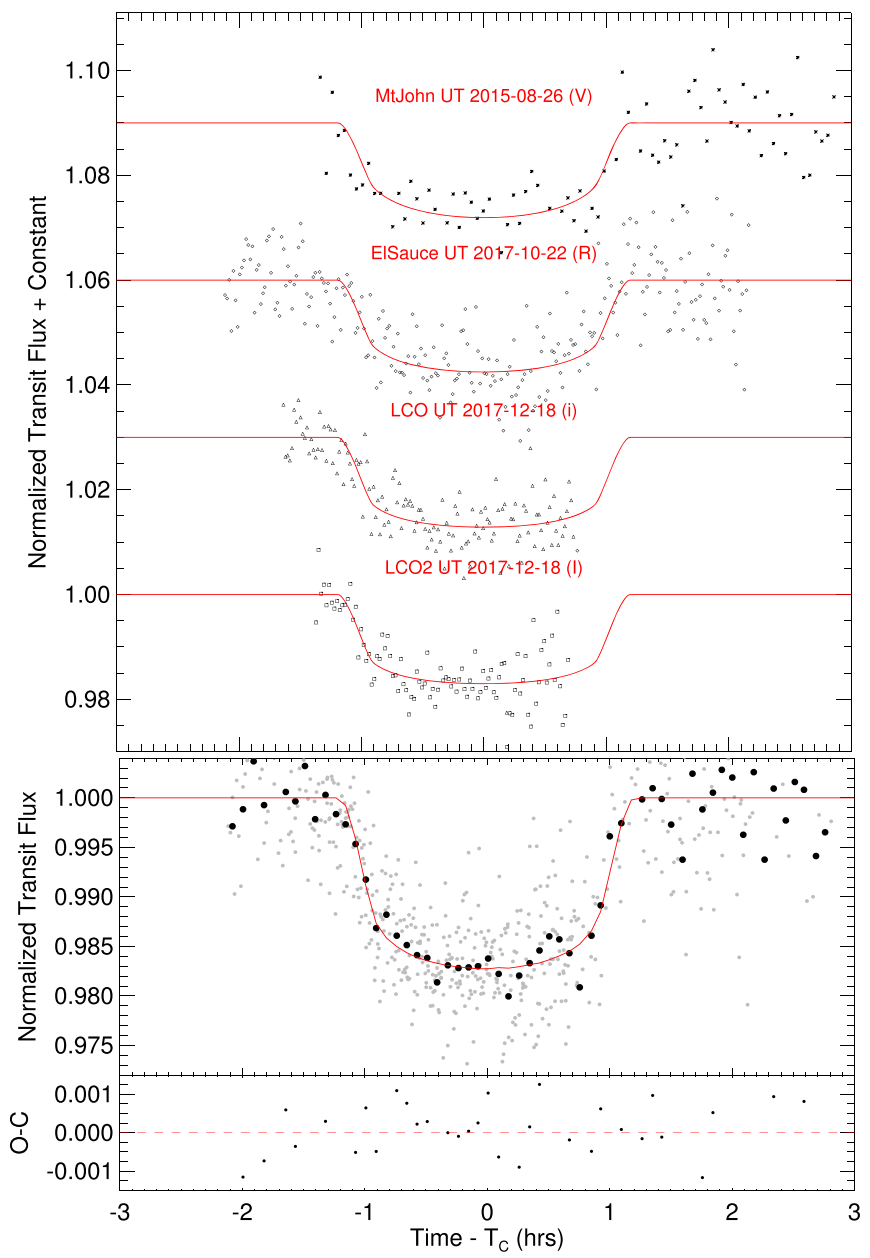

Figure 2. Top: transit light curves of KELT-22Ab from the KELT Follow-Up Network, after correcting for the contaminating flux from KELT-22B. The red line represents the best-fit model from the global fit in that photometric band. Each light curve is offset vertically by an arbitrary amount for clarity. Bottom: all follow-up transits combined into one light curve (gray) and a 5 minute binned light curve (black). The red line is the combined and binned models for each transit. We emphasize that the combined light curve is only for display purposes; the individual transit light curves were used in our analysis. The data used to create this figure are available.

KELT-22A was first observed at a low resolution ( $R \sim 3000)$ in the 3500-6000 $\AA$ range, allowing us to conclude that KELT-22A is a dwarf star suitable for further follow-up observations. We simultaneously find that KELT-22B is also a dwarf, but of a comparatively later spectral type. The system was then observed four more times at a medium resolution $(R \sim 7000)$ in the range of 5500-9000 $\AA$ for the purpose of measuring the radial velocity at a low precision sufficient for detecting an RV signal caused by a stellar-mass companion; however, we note that the radial velocity precision is insufficient to measure the RV signal caused by a planetarymass companion.

\subsubsection{TRES at FLWO}

We obtained 11 spectra of KELT-22A with the Tillinghast Reflector Échelle Spectrograph (TRES; Szentgyorgyi \& Fúrész 2007; Fúrész et al. 2008) on the $1.5 \mathrm{~m}$ telescope at the Fred Lawrence Whipple Observatory in Arizona. TRES is a fiber-fed échelle spectrograph (with a 2!"3 fiber), covering wavelengths between 3900 and $9100 \AA$, with a resolution of $R \sim 44000$. KELT-22A was observed with TRES 11 times between UT 2017 September 26 and UT 2017 November 22. From these spectra we determine the RV orbit of the system in the same manner as Buchhave et al. (2010). The wavelength solutions are derived from $\mathrm{Th}-\mathrm{Ar}$ hollow cathode lamp exposures that bracket each object spectrum. Radial velocities are obtained by cross-correlating multiple échelle orders of each spectrum, which are then weighted and combined, relative to the highest-quality observation, which is used as the template in the this analysis. The systemic velocity was derived from the single-order correlations of the $\mathrm{Mg} \mathrm{b}$ order against a library of synthetic spectra generated using the Kurucz (1992) atmosphere models and with the zero-point calculated using observations of IAU RV standard stars.

We also use these spectra to determine stellar properties, described in Section 3. Bisector spans (BSs) were also calculated, following the prescription of Buchhave et al. (2010).

\subsection{High-contrast Imaging}

As part of our standard process for validating transiting exoplanets, we observed KELT-22A with infrared highresolution adaptive optics (AO) imaging at Keck Observatory. The Keck Observatory observations were made with the NIRC2 instrument on Keck II behind the natural guide star AO system. The observations were made on UT 2017 December 07 in the narrowband $\mathrm{Br}-\gamma$ filter $\left(\lambda_{\circ}=2.1686 \mu \mathrm{m}\right.$, $\Delta \lambda=0.0326 \mu \mathrm{m})$ in the standard 3-point dither pattern that is used with NIRC2 to avoid the left lower quadrant of the detector, which is typically noisier than the other three quadrants. The dither pattern step size was $3^{\prime \prime}$ and was repeated three times, with each dither offset from the previous dither by 0 .'5. The observations utilized an integration time of 10 seconds with one co-add per frame for a total of 90 seconds. The camera was in the narrow-angle mode with a full field of view of $10^{\prime \prime}$ and a pixel scale of 0 ". $^{\prime \prime} 09942$ per pixel. We use the dithered images to remove sky background and dark current, and then align, flat-field, and stack the individual images. The NIRC2 AO data have a resolution of 0."049 (FWHM).

As noted in Section 2.2, there is a stellar companion to the southeast of the primary star that is easily detected in Keck imaging (see Figure 3), as well as from 2MASS imaging. The primary star's infrared 2MASS colors are consistent with the spectral type derived from the spectral energy distribution fit (Section 3.2) and the spectroscopic analysis that the star is a G2 main-sequence star (Section 3.1). The companion star is approximately 0.4 mag fainter than the primary star in the infrared and has colors that are consistent with the star being a late G-type or early K-type main-sequence star (see Figure 4). Given the location of the stars in the color-magnitude diagram, the companion star cannot be a background or foreground star that is highly attenuated by dust along the line of sight. As such, the companion star, KELT-22B, must be at a distance similar to that of KELT-22A. The Gaia DR2 parallax values for KELT-22A and KELT-22B are consistent to within the reported error, further evidencing their common distance.

The 2MASS, NIRC2, and Gaia imaging data span $\sim 20$ years, and all show consistent values for the projected separation (6". 1$)$ and position angle $\left(110^{\circ}\right)$ between KELT22A and KELT-22B. At a distance of $\sim 230$ pc (from Gaia, see 


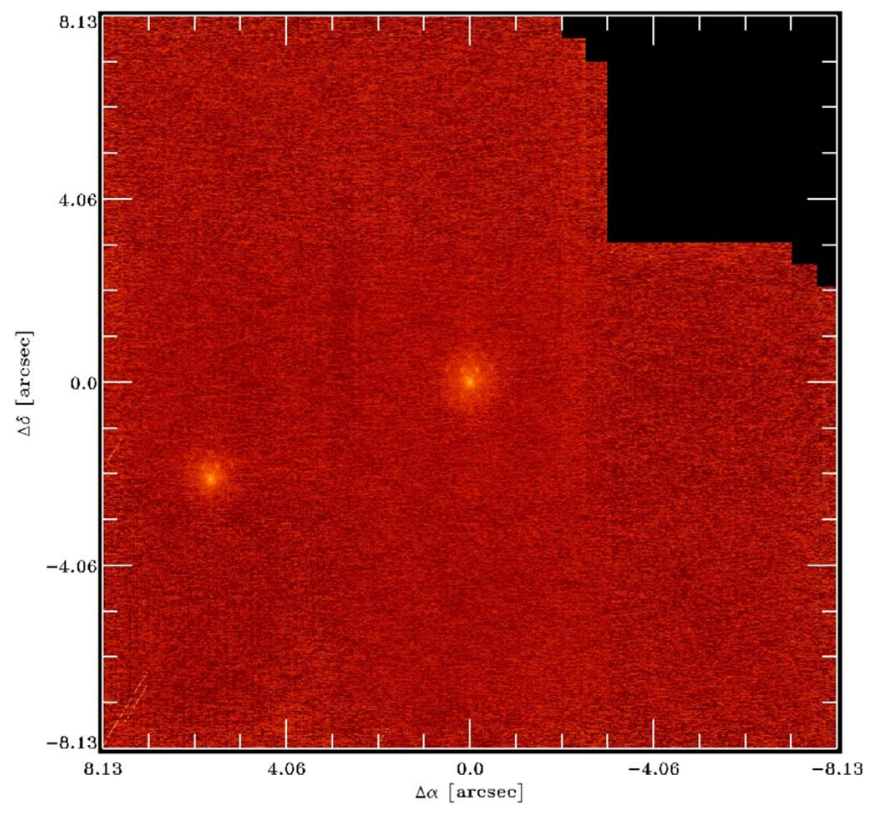

Figure 3. Keck II image from the NIRC2 instrument showing KELT-22A (center) and KELT-22B to the southeast. No other contaminating sources are evident.

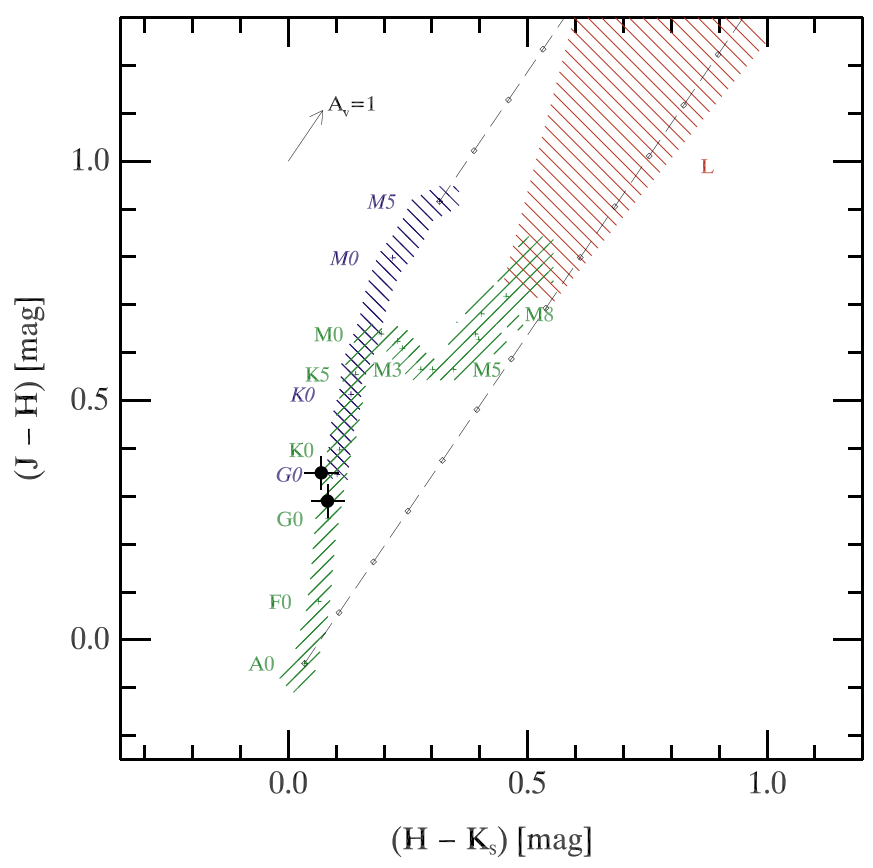

Figure 4. 2MASS color-color diagram for KELT-22A (lower black point) and KELT-22B (upper black point), as in Ciardi et al.(2011).The green hashed area denotes the main sequence, while the blue hashed area shows the giant branch. The red hashed region is where L-dwarfs are found. Diagonal lines trace the reddening zone for typical galactic interstellar extinction $(R=3.1)$. The SED analysis presented in Section 3.2 gives a low reddening value that is consistent with zero.

Table 1), the projected separation of the two stars is $\sim 1400 \mathrm{au}$, which corresponds to a $\sim 50,000$ year orbital period for the stars (assuming they are bound). This lies comfortably within the stellar companion orbital period distribution for nearby stars (Raghavan et al. 2010). At such a long orbital period, the projected orbital motion from the 1999 2MASS epoch to the 2017 Keck epoch should only be $\sim 0.04 \%$ of the entire orbit. The lack of any apparent relative motion is consistent with the
Table 1

Stellar Properties for KELT-22A

\begin{tabular}{|c|c|c|c|}
\hline \multirow[t]{4}{*}{ Other Names } & \multicolumn{3}{|l|}{ TYC 7518-468-1 } \\
\hline & \multicolumn{3}{|c|}{ 2MASS J23364036-3436404 } \\
\hline & \multicolumn{3}{|l|}{ TIC 77031414} \\
\hline & Description & Value & Ref. \\
\hline$\alpha_{\mathrm{J} 2000}$ & R.A. & $23^{\mathrm{h}} 36^{\mathrm{m}} 40^{\mathrm{s}} \cdot 325$ & 1 \\
\hline$\delta_{\mathrm{J} 2000}$ & Decl. & $-34^{\circ} 36^{\prime} 40^{\prime \prime} 42$ & 1 \\
\hline$N U V$ & GALEX NUV mag. & $16.810 \pm 0.020$ & 2 \\
\hline$B_{\mathrm{T}}$ & Tycho $B_{\mathrm{T}}$ mag. & $12.998 \pm 0.293$ & 1 \\
\hline$V_{\mathrm{T}}$ & Tycho $V_{\mathrm{T}}$ mag. & $11.314 \pm 0.097$ & 1 \\
\hline$B$ & $\begin{array}{l}\text { APASS Johnson } \\
\quad B \text { mag. }\end{array}$ & $11.838 \pm 0.011$ & $3^{\mathrm{a}}$ \\
\hline$V$ & $\begin{array}{l}\text { APASS Johnson } \\
\quad V \text { mag. }\end{array}$ & $11.102 \pm 0.034$ & $3^{\mathrm{a}}$ \\
\hline$g^{\prime}$ & APASS Sloan $g^{\prime}$ mag. & $11.426 \pm 0.014$ & $3^{\mathrm{a}}$ \\
\hline$r^{\prime}$ & APASS Sloan $r^{\prime}$ mag. & $10.913 \pm 0.036$ & $3^{\mathrm{a}}$ \\
\hline$i^{\prime}$ & APASS Sloan $i^{\prime}$ mag. & $10.792 \pm 0.061$ & $3^{\mathrm{a}}$ \\
\hline$J$ & 2MASS $J$ mag. & $10.374 \pm 0.050$ & 4 \\
\hline$H$ & 2MASS $H$ mag. & $10.084 \pm 0.070$ & 4 \\
\hline$K$ & 2MASS $K$ mag. & $10.002 \pm 0.050$ & 4 \\
\hline WISE1 & WISE1 mag. & $9.960 \pm 0.030$ & 5 \\
\hline WISE2 & WISE2 mag. & $9.980 \pm 0.028$ & 5 \\
\hline WISE3 & WISE3 mag. & $9.993 \pm 0.057$ & 5 \\
\hline WISE4 & WISE4 mag. & $\geqslant 8.456$ & 5 \\
\hline$\mu_{\alpha}$ & $\begin{array}{l}\text { Gaia DR2 proper } \\
\text { motion } \\
\text { in RA (mas } \mathrm{yr}^{-1} \text { ) }\end{array}$ & $87.963 \pm 0.061$ & 6 \\
\hline$\mu_{\delta}$ & $\begin{array}{l}\text { Gaia DR2 proper } \\
\text { motion } \\
\text { in } \operatorname{Dec}\left(\text { mas } \mathrm{yr}^{-1}\right)\end{array}$ & $-8.556 \pm 0.057$ & 6 \\
\hline$\Pi$ & $\begin{array}{l}\text { Gaia DR2 Paral- } \\
\quad \text { lax (mas) }\end{array}$ & $4.3456 \pm 0.0325$ & $6^{\mathrm{b}}$ \\
\hline$d$ & $\begin{array}{l}\text { Gaia-inferred dis- } \\
\quad \text { tance }(\mathrm{pc})\end{array}$ & $230.1 \pm 7.8$ & Section 3.4 \\
\hline$R V$ & $\begin{array}{l}\text { Systemic radial } \\
\text { velocity }\left(\mathrm{km} \mathrm{s}^{-1}\right)\end{array}$ & $-7.81 \pm 0.1$ & Section 3.1 .2 \\
\hline$v \sin I_{\star}$ & $\begin{array}{l}\text { Stellar rotational } \\
\text { velocity }\left(\mathrm{km} \mathrm{s}^{-1}\right)\end{array}$ & $7.9 \pm 0.5$ & Section 3.1 \\
\hline$T_{\text {eff }}$ & $\begin{array}{l}\text { Stellar effective } \\
\text { temperature }(\mathrm{K})\end{array}$ & $5767_{-49}^{+50}$ & Section 4.2 \\
\hline $\log g_{\star}$ & $\begin{array}{l}\text { Stellar surface grav- } \\
\text { ity (cgs) }\end{array}$ & $4.393_{-0.060}^{+0.039}$ & Section 4.2 \\
\hline$[\mathrm{m} / \mathrm{H}]$ & Stellar metallicity (dex) & $0.259_{-0.083}^{+0.085}$ & Section 4.2 \\
\hline Sp. Type & & G2 & Section 3.1 \\
\hline$A_{V}$ & Visual extinction (mag) & $0.00_{-0.00}^{+0.03}$ & Section 3.2 \\
\hline Age & Age (Gyr) & $1.5-5.0$ & Section 3.3 \\
\hline$U$ & Space motion $\left(\mathrm{km} \mathrm{s}^{-1}\right)$ & $-69.6 \pm 0.6$ & Section 3.4 \\
\hline$V$ & Space motion $\left(\mathrm{km} \mathrm{s}^{-1}\right)$ & $-33.9 \pm 0.3$ & Section 3.4 \\
\hline$W$ & Space motion $\left(\mathrm{km} \mathrm{s}^{-1}\right)$ & $-25.5 \pm 0.3$ & Section 3.4 \\
\hline
\end{tabular}

Notes. References are as follows: (1) Fabricius et al. (2002), (2) Bianchi et al. (2011), (3) Henden et al. (2015), (4) Cutri et al. (2003), (5) Cutri et al. (2013), (6) Gaia Collaboration et al. (2018) Gaia DR2 http://gea.esac.esa.int/archive, (7) Astraatmadja \& Bailer-Jones (2016), using the Milky Way model.

${ }^{a}$ APASS broadband photometry for KELT-22A is likely contaminated with flux from KELT-22B, as these values are systematically brighter than expected.

${ }^{\mathrm{b}}$ Value after correcting for the systematic offset of $-82 \mu$ as as described in Stassun \& Torres (2018).

two stars being bound. Indeed, Gaia DR2 proper motions for both components are the same to within $0.5 \mathrm{mas} \mathrm{yr}^{-1}$ (see Section 3.4). 


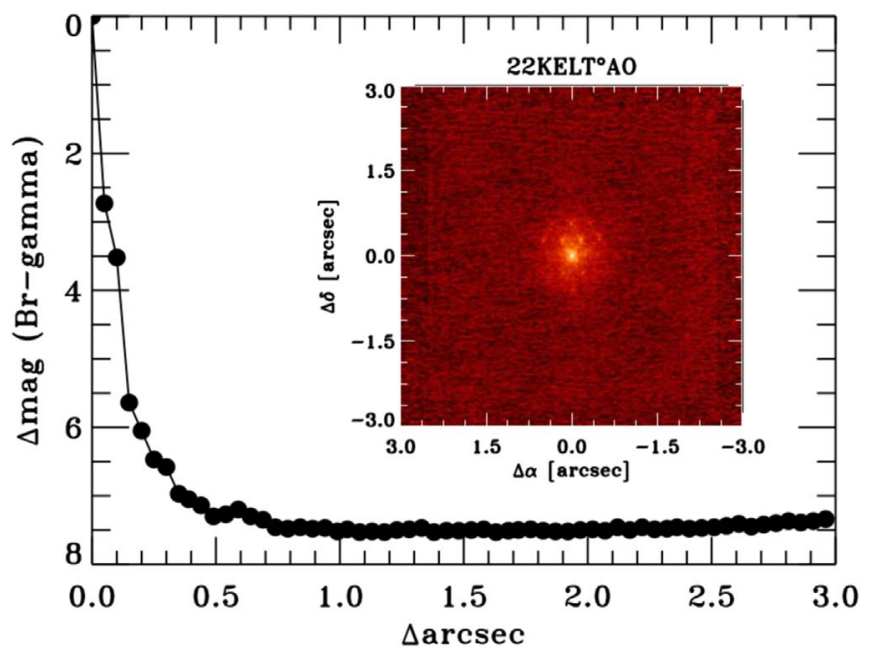

Figure 5. Contrast sensitivity at the $5 \sigma$ level and inset image of KELT-22A in $\mathrm{Br}-\gamma$ as observed with the NIRC2 camera at Keck Observatory. No additional sources are apparent within the limits of the data.

The AO imaging does not reveal any additional stellar components associated with KELT-22A, to within the limits of the data. The $5 \sigma$ sensitivities of the AO data shown in the contrast curve in Figure 5 were determined by injecting fake sources into the final combined images with separations from the primary targets in integer multiples of the central source's FWHM (Furlan et al. 2017; Ciardi et al. 2018; Siverd et al. 2018). No sources outside of $0 . ! 2$ are detected down to a contrast of $\Delta \operatorname{mag}_{\mathrm{Br}-\gamma}=6$, and nothing down to $\Delta$ $\operatorname{mag}_{\mathrm{Br}-\gamma}=8$ is detected past $\sim 0$ " 5 . We also note that only one set of lines is detected in our spectra of KELT-22A. Besides the neighbor at 6". 1 separation, there is no evidence for a close stellar companion to KELT-22A.

\section{Host Star Properties}

Table 1 lists various properties and measurements of KELT22A. These properties come from the literature and this work.

\subsection{Spectral Analysis}

We first obtained low-resolution and medium-resolution spectra of KELT-22A and KELT-22B to rule out the possibility of a stellar-mass companion causing the photometric signal. Detecting no large RV variations in either star, we then acquire spectra of KELT-22A at a higher resolution to measure the RV signal induced by the planet KELT-22Ab, and to estimate stellar properties.

\subsubsection{WiFeS Spectroscopy}

Our four WiFeS observations by which reconnaissance RVs were measured were spaced over the orbital period, as determined from the photometry. No RV signal was found above the uncertainty of a few $\mathrm{km} \mathrm{s}^{-1}$, ruling out the possibility of a stellar-mass companion orbiting KELT-22A at the photometric period. From the same exposures, RV measurements of KELT-22B were found to be flat within the measurement error. We also compare the absolute RV of both KELT-22A and its companion, and find them to be consistent within measurement errors, which is expected if they are bound. These absolute RV measurements from our four WiFeS spectra are in agreement with the systemic RV measured for
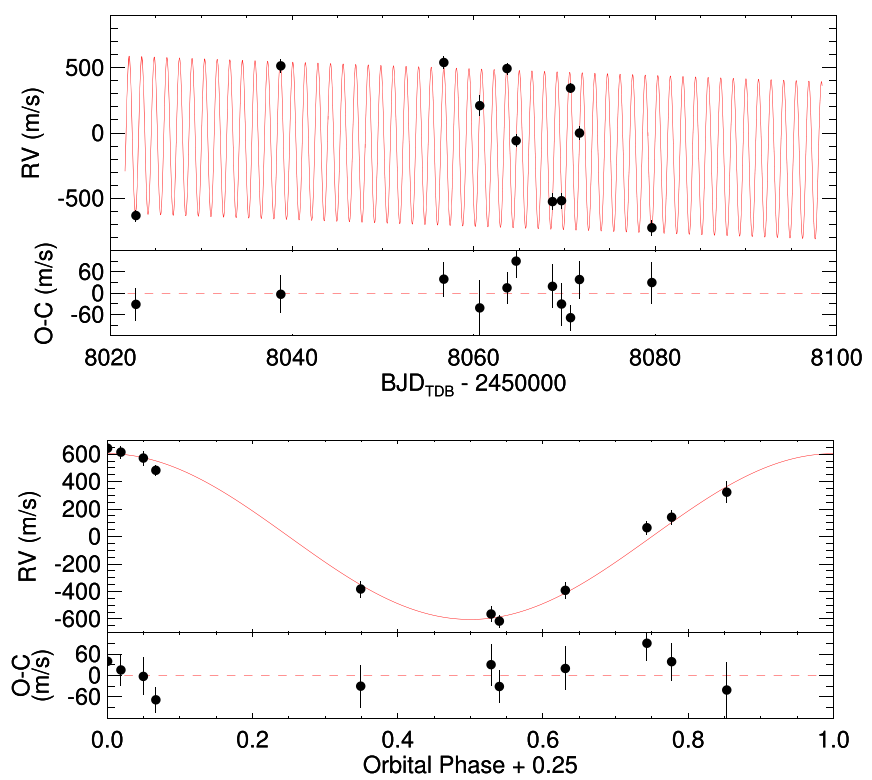

Figure 6. Top: the TRES RV measurements (black) of KELT-22A with the best-fit model shown in red. Bottom: the same measurements and model (after subtracting the linear trend and constant offset), phased to the orbital period.

Table 2

Relative Radial Velocity and Bisector Span Variation Measurements of KELT22A from TRES Spectra

\begin{tabular}{lccrc}
\hline \hline BJD & $\begin{array}{c}\mathrm{RV} \\
\left(\mathrm{m} \mathrm{s}^{-1}\right)\end{array}$ & $\begin{array}{c}\sigma_{\mathrm{RV}} \\
\left(\mathrm{m} \mathrm{s}^{-1}\right)\end{array}$ & $\begin{array}{c}\mathrm{BS} \\
\left(\mathrm{m} \mathrm{s}^{-1}\right)\end{array}$ & $\begin{array}{c}\sigma_{\mathrm{BS}} \\
\left(\mathrm{m} \mathrm{s}^{-1}\right)\end{array}$ \\
\hline 2458022.80169 & -632 & 45 & 53 & 23 \\
2458038.76176 & 515 & 52 & 87 & 30 \\
2458056.72000 & 541 & 48 & -14 & 31 \\
2458060.67554 & 210 & 77 & -230 & 49 \\
2458063.67870 & 494 & 44 & -89 & 33 \\
2458064.68310 & -59 & 47 & -42 & 57 \\
2458068.68726 & -525 & 60 & 89 & 35 \\
2458069.68318 & -518 & 58 & 52 & 28 \\
2458070.67870 & 344 & 36 & 38 & 37 \\
2458071.66350 & 0.0 & 52 & 60 & 28 \\
2458079.63865 & -725 & 57 & -4 & 24 \\
\hline
\end{tabular}

KELT-22A from the higher-resolution spectra described in Section 3.1.2.

\subsubsection{TRES Spectroscopy}

We estimate some physical properties of KELT-22A using the TRES spectra. These spectra were analyzed using the Stellar Parameter Classification (SPC) procedure of Buchhave et al. (2012). We run the SPC procedure without fixing any parameters, and take the error-weighted mean value for each stellar parameter. This gives a value for the effective temperature of $T_{\text {eff }}=5771 \pm 50 \mathrm{~K}$, a surface gravity of $\log g_{\star}$ $=4.49 \pm 0.1 \mathrm{~cm} \mathrm{~s}^{-2}$, metallicity of $[\mathrm{m} / \mathrm{H}]=0.26 \pm 0.08$, and a projected equatorial rotational velocity of $v \sin I_{\star}$ $=7.9 \pm 0.5 \mathrm{~km} \mathrm{~s}^{-1}$. This is consistent with a spectral type of $\mathrm{G} 2 \mathrm{~V}$.

Our RV measurements from TRES are reported in Table 2 along with the corresponding BSs, which are included as part of the false-positive analysis presented in Section 5. These RVs are consistent with the ephemeris determined from our photometric data and are plotted in the upper panel of Figure 6, along with the best-fit model (after subtracting off a 


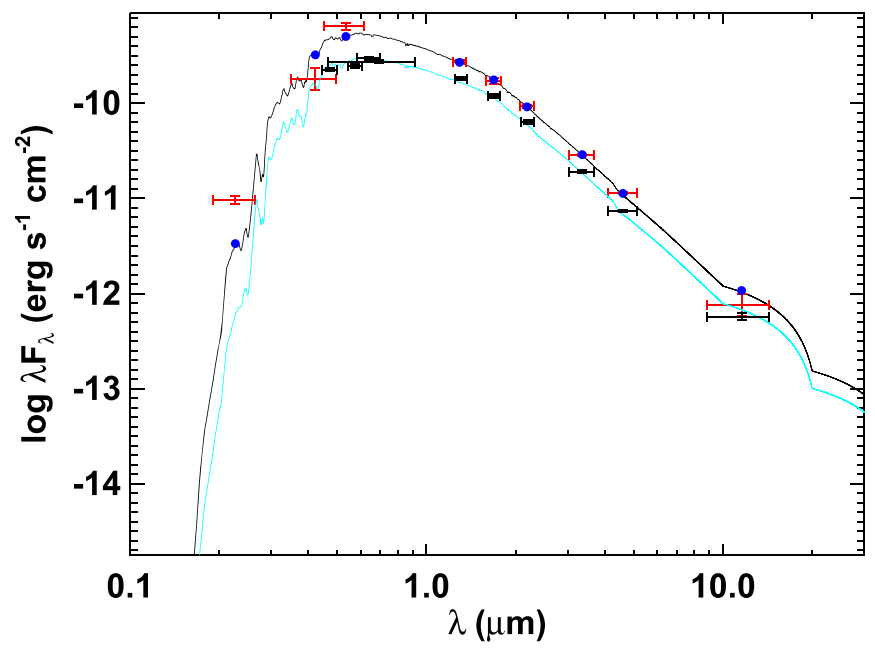

Figure 7. SED fit for KELT-22A (black curve) and the neighboring star, KELT-22B (cyan curve), showing the best-fit stellar atmospheric models. The red and black crosses show the photometric values and their errors for KELT22A and KELT-22B, respectively. These values for KELT-22A are listed in Table 1 . The blue points are the predicted integrated fluxes at the corresponding passbands for KELT-22A.

constant offset and a linear trend), with residuals shown immediately below. The RV semi-amplitude is $K=592 \pm 20$ $\mathrm{m} \mathrm{s}^{-1}$. In addition to the oscillatory RV signal in KELT-22A caused by the planetary orbit, there is evidence for a linear trend of $-2.06_{-0.91}^{+0.93} \mathrm{~m} \mathrm{~s}^{-1}$ day $^{-1}$ at low significance. From the $\mathrm{RV}$ signal, we find a minimum mass for the planet of $M_{P} \sin i$ $=3.46_{-0.14}^{+0.15} M_{J}$. RV data phased to the orbital period and the best-fit model and residuals are shown in the lower panel of Figure 6. The systemic RV of the KELT-22A system is $-7.81 \pm 0.1 \mathrm{~km} \mathrm{~s}^{-1}$.

\subsection{SED Analysis}

We performed a fit to the broadband spectral energy distribution (SED) of KELT-22A as an additional constraint on the stellar parameters in the global system fit. We assembled the available broadband photometry from the literature (Table 1), with measurements spanning over the wavelength range $0.2-10 \mu \mathrm{m}$ (Figure 7). The high-resolution imaging (see Section 2.4) confirms a nearby companion star that is sufficiently well separated from KELT-22A that it should not contaminate the broadband photometry from most catalogs. We were careful to use only broadband flux values from catalogs where these two sources are clearly resolved. For example, APASS photometry for KELT-22A is likely contaminated by flux from KELT-22B, and is therefore not included in the SED fit. We fit SEDs to both components, assuming (for the purposes of the fit) the same $A_{V}$ and distance for both.

We fitted the SED numerically in multiple steps. First, we fit a single Kurucz stellar atmosphere model (Kurucz 1992) to the broadband photometry of the bright primary star using the spectroscopic parameters $T_{\text {eff }}, \log g_{\star}$, and $[\mathrm{Fe} / \mathrm{H}]$ from Section 3.1 as an initial guess, and the distance inferred from the measured Gaia parallax. The free parameters of the fit were the reddening $\left(A_{V}\right)$ and the overall flux normalization. The reddening used a standard Cardelli interstellar reddening law (Cardelli et al. 1989) applied to the stellar model, restricted to the maximum permitted value to the full line of sight extinction from Schlegel et al. (1998). The model bandpass-integrated fluxes in each band were compared to the observed fluxes, and $\chi^{2}$ was calculated based on the observed photometric errors. Next, we introduced a second Kurucz stellar model for KELT$22 \mathrm{~B}$. We used the same procedure, except we allowed $T_{\text {eff }}$ for KELT-22B to be a free parameter. We iterated the fit of KELT22B with the fit of KELT-22A, adjusting the $A_{V}$ between each iteration until the best fits for both stars shared the same $A_{V}$. The final $\chi^{2}$ represents the goodness of fit of all of the measurements (for both stars) and accounting for the number of free fit parameters.

The best fit is shown in Figure 7, and ignoring the GALEX NUV passband that shows an excess indicative of moderate chromospheric activity, ${ }^{44}$ it has a reduced $\chi^{2}$ of 3.3. We find for KELT-22A a best fit $A_{V}=0.00_{-0.00}^{+0.03}$. We can integrate the best-fit SED to obtain the (unextincted) bolometric flux at Earth: $F_{\text {bol }}=6.73_{-0.45}^{+0.48} \times 10^{-10} \mathrm{erg} \mathrm{s}^{-1} \mathrm{~cm}^{-2}$. With the Gaia DR2 parallax and the adopted $T_{\text {eff }}$, this then allows a measure of the stellar radius, including the $-82 \mu$ as offset in the Gaia DR2 parallax suggested in the literature (see, e.g., Stassun \& Torres 2018): $R_{\star}=1.04 \pm 0.04 R_{\odot}$. This empirical $R_{\star}$ is consistent with the value found from the global model described in Section 4.2.

Finally, we use our two-component SED fit (Figure 7) to assess the degree to which our KELT-22A planet transit model is affected by flux contamination from the neighboring companion, considering that both stars are contained within a single KELT pixel, and all of our follow-up photometry was reduced using an aperture that includes both stars. Taking the ratio of fluxes in each bandpass for which we have transit observations, we obtain the following flux ratios $\left(f_{B} / f_{A}\right)$ : $f_{V}=0.54, f_{R_{C}}=0.56, f_{I_{C}}=0.58$, and $f_{i}=0.57$. These flux ratios are then used to correct the observed transit depths in the global system fitting below, as in, e.g., Ciardi et al. (2015).

\subsection{Stellar Models and Age}

We can use the properties of KELT-22A to estimate its age as inferred from stellar evolution models. In Figure 8, we represent the $T_{\text {eff }}$ and $\log g_{\star}$ of KELT-22A relative to the predicted evolutionary track of a star with the mass and metallicity of KELT-22A. These parameters are derived from our global fit in Section 4.2). The Yonsei-Yale models (YY; Yi et al. 2001) are used to compute an evolutionary track for a solar-mass star, which is a reasonable estimate of the mass of KELT-22A as determined from our global fit (see Section 4.2). The location of KELT-22A on this evolutionary track suggests that the star is still early in its main-sequence lifetime, and thus its slow evolution translates to a relatively large uncertainty on the age. Specifically, we infer an approximate age for KELT22A of 1.5-5.0 Gyr.

Comparison to the MESA Isochrones and Stellar Tracks (MIST) project (Choi et al. 2016; Dotter 2016) isochrones in the analysis of Section 6.3 yields an age of $2.6 \mathrm{Gyr}$, using solar parameters and forcing the radius and density to be that found from the global fit, in general agreement with the above analysis. Another indication of youth is that KELT-22A appears to have a super-solar metallicity, with $[\mathrm{m} / \mathrm{H}] \sim 0.26$, which is more typical of a relatively young (disk) star.

KELT-22A has a rotation rate that is unusually high for a star of this spectral type. This is best evidenced by its high

\footnotetext{
44 We note that we cannot rule out that the GALEX NUV excess originates from the close stellar companion. However, the observed excess is also consistent with the rapid rotation observed for KELT-22A and with the relatively unevolved age of the star (see Section 3.3).
} 


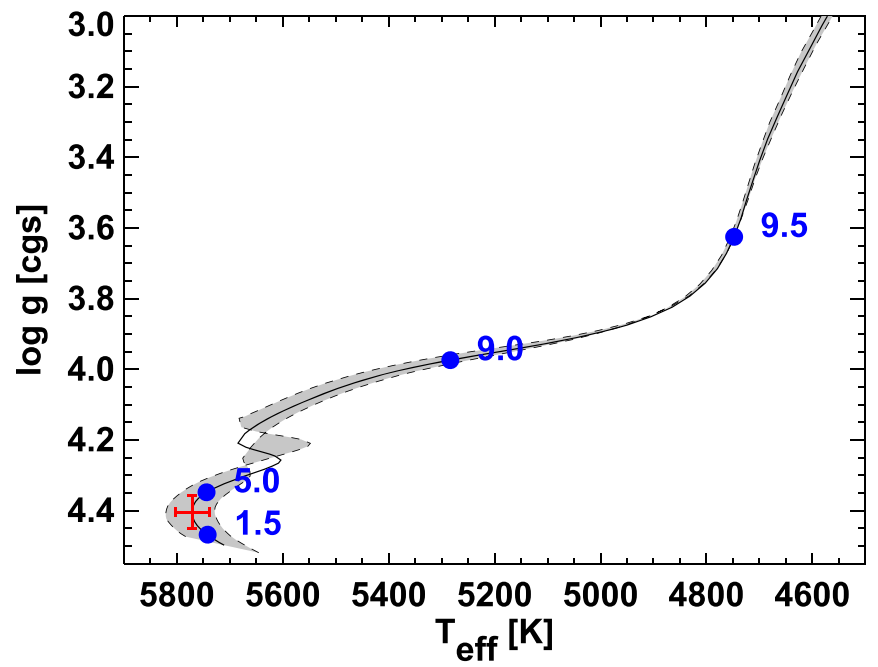

Figure 8. Evolution of the KELT-22A system in the Kiel diagram. The red cross represents the KELT-22A parameters from the final global fit. The black curve represents the theoretical evolutionary track for a star with the mass and metallicity of KELT-22A, and the gray swath represents the uncertainty on that track based on the uncertainties in mass and metallicity. Nominal ages in Gyr are shown as blue dots.

$v \sin I_{\star}=7.9 \pm 0.5 \mathrm{~km} \mathrm{~s}^{-1}$, measured from TRES spectra. Other circumstantial evidence consistent with rapid rotation is the NUV excess in the SED fit, which is indicative of chromospheric activity (being higher in more rapidly rotating stars), and an $\sim 8$ day sinusoidal signal in the KELT photometry, which could be caused by rotational modulation (see Section 6.2). Such rapid rotation may suggest a young star that has not yet had time to spin down due to magnetic braking. Another possible explanation for the rapid rotation is that tidal interactions between the star and planet are acting (or have acted) to spin the star up. This latter possibility is discussed further in Section 6.3.

\subsection{Location in the Galaxy, UVW Space Motion, Galactic Population}

KELT-22A is located at $\alpha_{\mathrm{J} 2000}=23^{\mathrm{h}} 36^{\mathrm{m}} 40.325$ and $\delta_{\mathrm{J} 2000}=$ $-34^{\circ} 36^{\prime} 40^{\prime \prime} 42$, which corresponds to Galactic coordinates of $\ell=3^{\circ} .3$ and $b=-72^{\circ} .3$. Given the Stassun \& Torres (2018) corrected Gaia DR2 parallax of $4.3456 \pm 0.0325$ mas, this implies a distance of $230.1 \pm 7.8 \mathrm{pc}$, ignoring Lutz-Kelker bias (Lutz \& Kelker 1973). Therefore, KELT-22A is $~ 219$ pc below the Galactic plane.

The kinematics of KELT-22A in the Galaxy are even more intriguing than its spatial location. Given the Gaia DR2 proper motions of $\left(\mu_{\alpha}, \mu_{\delta}\right)=(87.963 \pm 0.061,-8.556 \pm$ 0.057) mas $\mathrm{yr}^{-1}$, the Gaia parallax, and the absolute radial velocity as determined from the TRES spectroscopy of $-7.81 \pm 0.1 \mathrm{~km} \mathrm{~s}^{-1}$, we find that KELT-22A has a threedimensional Galactic space motion of $(U, V, W)=(-69.6 \pm$ $0.6,-33.9 \pm 0.3,-25.5 \pm 0.3) \mathrm{km} \mathrm{s}^{-1}$, where positive $U$ is in the direction of the Galactic center, and we have adopted the Coşkunoğlu et al. (2011) determination of the solar motion with respect to the local standard of rest. These values yield a $18.3 \%$ probability that KELT-22A is a thick-disk star, according to the classification scheme of Bensby et al. (2003). This is roughly an order of magnitude larger than the probability of any other KELT discovery being a thick-disk star according to the criteria given in Bensby et al. (2003).

To give these kinematics additional context, we computed the Galactic orbit of KELT-22A using the galpy ${ }^{45}$ package (Bovy 2015). We use our knowledge of the system's threedimensional spatial location and its three-dimensional Galactic space motion, along with the "MWPotential2014" Galactic potential (which approximately simulates that of the Milky Way) in galpy, and compute the motion of KELT-22A through the Galaxy from now until 2 Gyr in the future. These results are shown in Figure 9 (with the predicted path of the Sun shown for comparison). Figure 9 demonstrates that the Galactic orbit of KELT-22A is eccentric ( $e=0.31)$, covers a large range in Galactocentric distance (from 4.7-9.1 kpc from the Galactic center), and reaches up to $\sim 280 \mathrm{pc}$ above the Galactic midplane. Stars of KELT-22A's spectral type (G2V) have a typical scale height of $z_{d} \sim 123 \pm 20$ pc (Bovy 2017).

We adopt the Gaia DR2 parallax and proper motion information, since these measurements are by far the most precise available. Gaia DR2 includes both stellar components of KELT-22, with parallax values that are consistent within errors, and proper motions that are essentially the same, differing by $\lesssim 0.5 \mathrm{mas} \mathrm{yr}^{-1}$. This supports our claim that KELT-22A and KELT-22B are two components of a bound visual binary system. Gaia DR1 lists measurements for KELT-22A that are consistent with DR2, but does not include KELT-22B. We note that the Southern Proper Motion Program (SPM2; Platais et al.

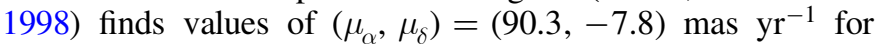
KELT-22A, in agreement with Gaia DR2. However, other catalogs list values that are significantly smaller, including Tycho-2 (Høg et al. 2000) and PPMX (which builds off of Tycho-2; Röser et al. 2008). UCAC4 (Zacharias et al. 2012) includes proper motion measurements for both KELT-22A and its companion, listing $\left(\mu_{\alpha}, \mu_{\delta}\right)=(43.0,6.7){\text { mas } \mathrm{yr}^{-1} \text { and }}^{-1}$ $\left(\mu_{\alpha}, \mu_{\delta}\right)=(113.7,-827.5)$ mas $\mathrm{yr}^{-1}$, respectively. We adopt the values from Gaia DR2, and justify this choice by drawing attention to the consistency among SPM2, UCAC2, NOMAD, XPM, SPM4, APOP, and UCAC5.

\section{Results \\ 4.1. Light Curve Detrending and Deblending}

Because KELT-22A and KELT-22B are not fully resolved in the follow-up light curves, an aperture is placed around both sources when extracting photometry. We must then account for the flux from KELT-22B to accurately calculate the planetary radius. Without deblending, the planetary radius will be significantly underestimated. The flux ratios in each bandpass for which we have a follow-up light curve of sufficient quality are calculated in Section 3.2, and are then incorporated into the global model. Each light curve used in the fit includes airmass as a detrending parameter.

\subsection{Global Model Results}

Using a modified version of EXOFAST (Eastman et al. 2013) that we hereafter refer to as MULTIFAST, we simultaneously fit the follow-up time-series photometry and TRES RVs to properly determine the global parameters of the KELT-22A system. MULTIFAST is an IDL-based fitting tool that runs simultaneous Markov chain Monte Carlo (MCMC)

\footnotetext{
45 http://github.com/jobovy/galpy
} 

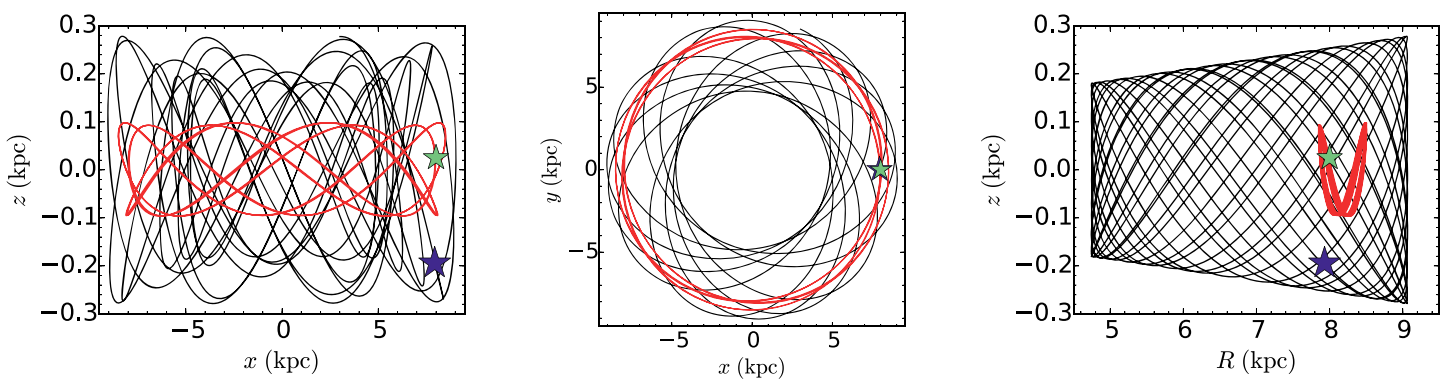

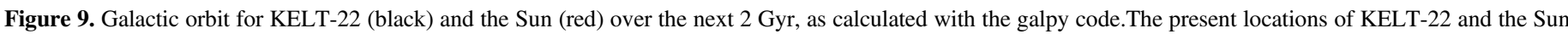
are indicated by purple and green star symbols, respectively.

analyses to determine each parameter's posterior probability distribution using a Keplerian model and allowing for a linear trend. From our analysis of the TRES spectra, we enforce a Gaussian prior on $T_{\text {eff }}$ and $[\mathrm{Fe} / \mathrm{H}]$ while setting a starting point for $\log g_{\star}$. We do not enforce a prior on $\log g_{\star}$ because it is expected to be better constrained from fitting the light curves (Seager \& Mallén-Ornelas 2003; Mortier et al. 2014). The KELT light curve (see Figure 1) is not included in the global fit, but we set Gaussian priors on $\mathrm{T}_{C}$ and the period from our analysis of the KELT data. To get these priors, we run a MULTIFAST fit on the KELT light curve using the determined box-least squares (BLS) parameters as starting points. We use the YY isochrones or the empirical Torres relations (Torres et al. 2010) within the global fit to constrain $R_{\star}$ and $M_{\star}$. We use the flux ratios found in Section 3.2 to correct each light curve by accounting for the contaminating flux from the stellar companion to KELT-22A. Our implementation of MULTIFAST does not explicitly fit for jitter terms for the TRES RV points, but rather scales the errors to properly represent them (as in Siverd et al. 2012). No boundaries are given to any of the priors or fitted parameters. We run separate YY and Torres global fits where the eccentricity is assumed to be zero. We also run separate YY and Torres fits where we fit for the eccentricity. The results of all four fits are consistent to $1 \sigma$ and are shown in Tables 3 and 4. For the analysis and discussion in this paper, we (somewhat arbitrarily) adopt the YY circular fit results (column two in Tables 3 and 4). The quadratic limb-darkening parameters included in Tables 3 and 4 are derived by interpolating the Claret \& Bloemen (2011) tables with each step in $\log g_{\star}, T_{\text {eff }}$, and $[\mathrm{Fe} / \mathrm{H}]$, and are not explicitly fit. We do not conduct a transit timing variation analysis, because we do not have enough high-quality observations that capture the full event to make such an analysis worthwhile.

\section{False-positive Analysis}

Many different methods were used to test various falsepositive scenarios. KELT-22A and KELT-22B are partially resolved in our photometric follow-up observations. For the photometric observations used in the global fit, in all cases careful placement of apertures reveals that KELT-22A does in fact have a transit event, while no such variability is apparent in KELT-22B. This strongly suggests that the detected photometric variability is in KELT-22A, and not its companion. Our $\mathrm{RV}$ data, in which the two sources are clearly resolved, corroborate this by revealing an RV signal in KELT-22A that is consistent with the reflex motion caused by a planetary orbit. Furthermore, our AO observations reveal no additional stellar

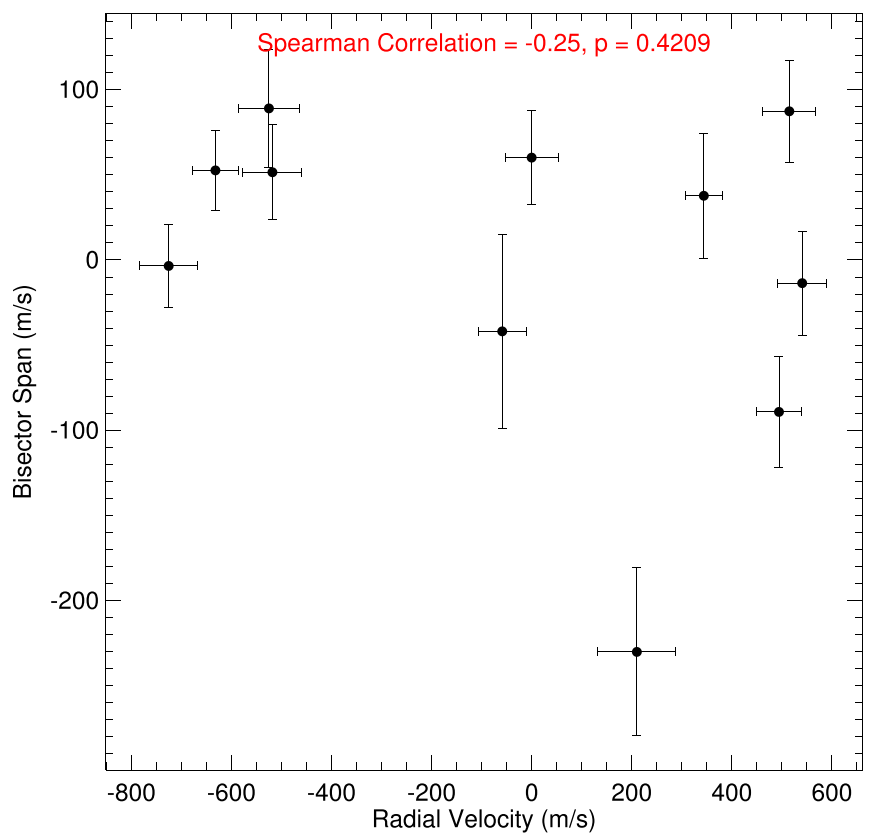

Figure 10. Bisector spans for the TRES RV spectra for KELT-22A plotted against the RV values. We find no correlation between these quantities.

objects, and there is nothing to suggest a second set of lines in our TRES spectra.

Our follow-up light curves cover the $V, R, I$, and $i$ ' passbands, and all show a consistent depth. EBs and blended EBs typically produce depths that are chromatic across this range of filters (O'Donovan et al. 2006). While the achromatic nature of the measured transit depth in the light curves of KELT-22A does not absolutely rule out the possibility of an EB or blended EB, it is consistent with a planetary scenario.

To further test the planetary hypothesis, we examine the RV bisector spans using the procedure of Buchhave et al. (2010). If the observed variability is caused by an unresolved EB, a correlation between the BSs and the measured RV values is expected due to the line-profile asymmetries that a blended EB will produce through its orbital motion (Torres et al. 2004). We find no correlation between the RV values and the BSs (see Figure 10), suggesting that the RV variation is caused by genuine orbital motion of KELT-22A.

Stellar activity, such as starspots, can sometimes mimic the signatures of exoplanets (e.g., Desort et al. 2007). However, this effect tends to cause variability at the stellar rotation period, which is significantly longer than the orbital period of KELT-22Ab (Section 6.2), and is also likely to impart 
Table 3

Median Values and 68\% Confidence Intervals for the Physical and Orbital Parameters of the KELT-22 System

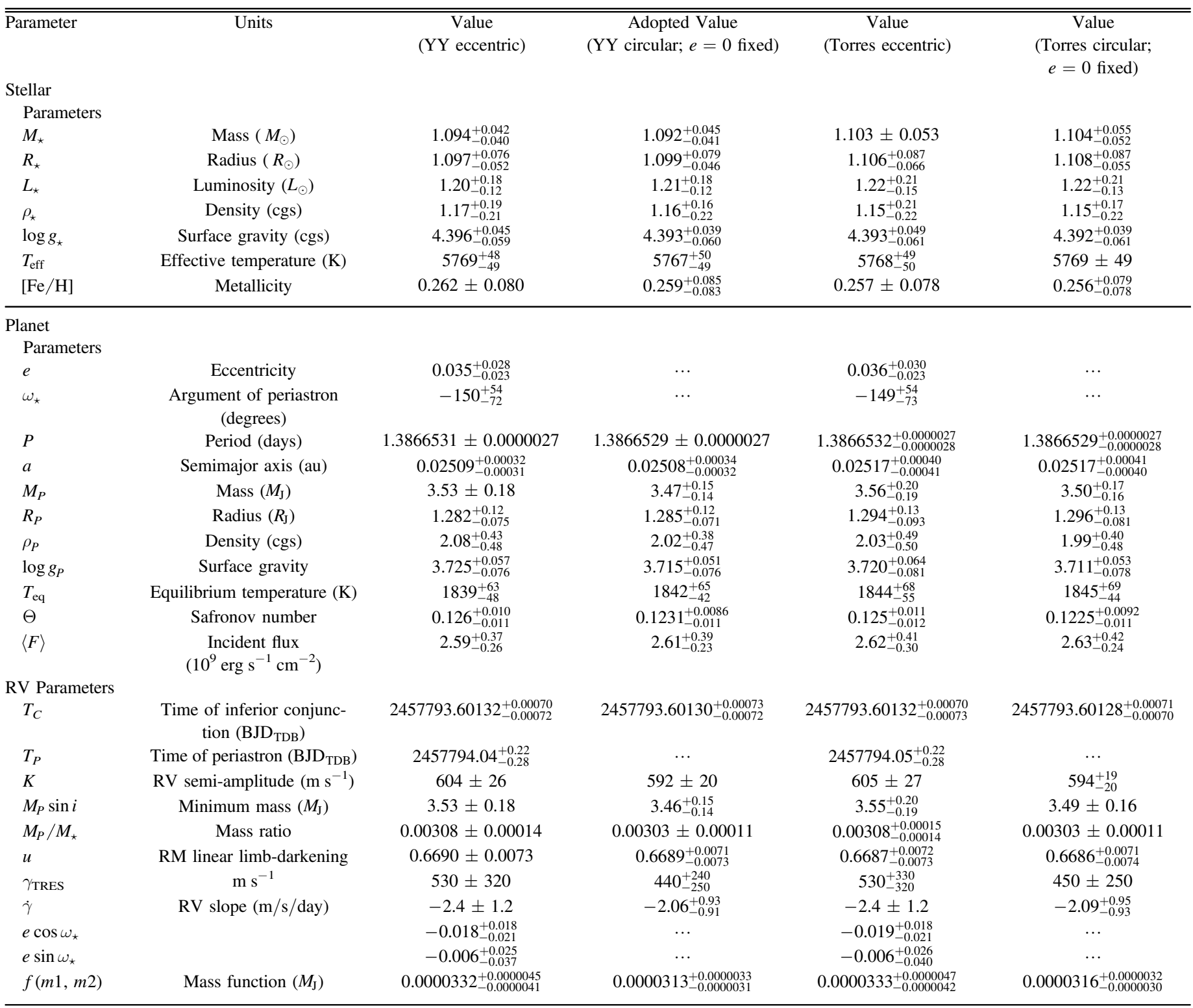

Note. The $\gamma_{\text {TRES }}$ values are the offset for the arbitrary zero-point of the relative velocities that result from the choice of observation used for the template. The planetary equilibrium temperature, $T_{\mathrm{eq}}$, was calculated assuming zero albedo and complete heat redistribution.

correlations between the BSs and RVs, which we find no evidence for.

Having ruled out all of the reasonable false-positive scenarios, we are confident that the photometric transit and the RV variability are intrinsic to KELT-22A, and are caused by a transiting exoplanet.

\section{Discussion}

\subsection{Stellar Multiplicity and the Possibility of a Second Planet}

The role of binarity enriches and complicates the formation and evolution of exoplanet systems. Planetary systems are found in various binary configurations ( $\mathrm{Ngo}$ et al. 2016), and even in hierarchical triple systems (e.g., Eastman et al. 2016). Our analysis of KELT-22A and KELT-22B suggest they are bound (Section 2.4). The discovery of KELT-22Ab adds to the population of transiting short-period gas giants with widely separated stellar companions.

At the projected separations of KELT-22A and KELT-22B $(\sim 6$ !' 1$)$, which are nearly equal mass, the expected radial velocity amplitude of the primary star in this wide binary is, assuming the system is edge-on, $\sim 0.5-1 \mathrm{~km} \mathrm{~s}^{-1}$, depending on the eccentricity of the orbit. In addition to the RV signal caused by the orbit of KELT-22Ab, we detect a linear slope of $-2.06_{-0.91}^{+0.93} \mathrm{~m} \mathrm{~s}^{-1}$ day $^{-1}$ in our RV data for KELT-22A at a somewhat low significance. The time span of the radial velocity observations is only 80 days long and is thus a very small fraction of the entire binary orbit. Even at its steepest, the expected linear slope of the KELT-22A RVs caused by KELT22B is on the order of $10^{-4} \mathrm{~m} \mathrm{~s}^{-1} \mathrm{~d}^{-1}$, which is significantly smaller than the trend seen in the RVs.

Using Equation (2) of Bowler (2016) and our measured RV trend, we find that the tertiary mass must be at least 2.8, 11.1, 
Table 4

Median Values and 68\% Confidence Interval for the Physical and Orbital Parameters of the KELT-22 System

\begin{tabular}{|c|c|c|c|c|c|}
\hline Parameter & Units & $\begin{array}{c}\text { Value } \\
\text { (YY eccentric) }\end{array}$ & $\begin{array}{c}\text { Adopted Value } \\
\text { (YY circular; } e=0 \text { fixed) }\end{array}$ & $\begin{array}{c}\text { Value } \\
\text { (Torres eccentric) }\end{array}$ & $\begin{array}{c}\text { Value } \\
\text { (Torres circular; } e=0 \text { fixed) }\end{array}$ \\
\hline \multicolumn{6}{|c|}{ Primary Transit } \\
\hline$R_{P} / R_{\star}$ & $\begin{array}{l}\text { Radius of the planet in } \\
\text { stellar radii }\end{array}$ & $0.1204_{-0.0028}^{+0.0031}$ & $0.1203_{-0.0028}^{+0.0032}$ & $0.1205_{-0.0029}^{+0.0032}$ & $0.1204_{-0.0027}^{+0.0031}$ \\
\hline$a / R_{\star}$ & $\begin{array}{l}\text { Semimajor axis in stellar } \\
\text { radii }\end{array}$ & $4.92_{-0.32}^{+0.25}$ & $4.91_{-0.33}^{+0.21}$ & $4.90_{-0.34}^{+0.29}$ & $4.89_{-0.34}^{+0.22}$ \\
\hline$i$ & Inclination (degrees) & $86.4_{-2.2}^{+2.3}$ & $86.5_{-2.3}^{+2.2}$ & $86.3_{-2.2}^{+2.4}$ & $86.3_{-2.3}^{+2.4}$ \\
\hline$b$ & Impact parameter & $0.31_{-0.19}^{+0.17}$ & $0.30_{-0.19}^{+0.16}$ & $0.32_{-0.20}^{+0.16}$ & $0.31_{-0.20}^{+0.16}$ \\
\hline$\delta$ & Transit depth & $0.01451_{-0.00068}^{+0.00077}$ & $0.01448_{-0.00067}^{+0.00078}$ & $0.01452_{-0.00068}^{+0.00079}$ & $0.01450_{-0.00065}^{+0.00076}$ \\
\hline$T_{\mathrm{FWHM}}$ & FWHM duration (days) & $0.0863_{-0.0017}^{+0.0016}$ & $0.0862 \pm 0.0016$ & $0.0863_{-0.0017}^{+0.0016}$ & $0.0862 \pm 0.0017$ \\
\hline$\tau$ & $\begin{array}{l}\text { Ingress/egress dura- } \\
\text { tion (days) }\end{array}$ & $0.0116_{-0.0010}^{+0.0023}$ & $0.0116_{-0.0010}^{+0.0021}$ & $0.0118_{-0.0012}^{+0.0024}$ & $0.0117_{-0.0011}^{+0.0022}$ \\
\hline$T_{14}$ & Total duration (days) & $0.0983_{-0.0022}^{+0.0025}$ & $0.0981_{-0.0022}^{+0.0025}$ & $0.0984_{-0.0023}^{+0.0026}$ & $0.0982_{-0.0023}^{+0.0025}$ \\
\hline$T_{C, 0}$ & Mid-transit time $\left(\mathrm{BJD}_{\mathrm{TDB}}\right)$ & $2457261.1265 \pm 0.0013$ & $2457261.1266_{-0.0013}^{+0.0014}$ & $2457261.1265_{-0.0013}^{+0.0014}$ & $2457261.1265 \pm 0.0013$ \\
\hline$T_{C, 1}$ & Mid-transit time (BJD $\left.{ }_{\mathrm{TDB}}\right)$ & $2458048.74549_{-0.00083}^{+0.00081}$ & $2458048.74546_{-0.00084}^{+0.00078}$ & $2458048.74549_{-0.00084}^{+0.00079}$ & $2458048.74542 \pm 0.00081$ \\
\hline$T_{C, 2}$ & Mid-transit time $\left(\mathrm{BJD}_{\mathrm{TDB}}\right)$ & $2458105.59827_{-0.00089}^{+0.00087}$ & $2458105.59824_{-0.00090}^{+0.00084}$ & $2458105.59828_{-0.00090}^{+0.00085}$ & $2458105.59820_{-0.00089}^{+0.00087}$ \\
\hline$T_{C, 3}$ & Mid-transit time $\left(\mathrm{BJD}_{\mathrm{TDB}}\right)$ & $2458105.59827_{-0.00089}^{+0.00087}$ & $2458105.59824_{-0.00090}^{+0.00084}$ & $2458105.59828_{-0.00090}^{+0.00085}$ & $2458105.59820_{-0.00089}^{+0.00087}$ \\
\hline$u_{1 I}$ & Linear Limb-darkening & $0.2990_{-0.0085}^{+0.0088}$ & $0.2988_{-0.0083}^{+0.0087}$ & $0.2988 \pm 0.0086$ & $0.2985_{-0.0086}^{+0.0085}$ \\
\hline$u_{2 I}$ & Quadratic Limb-darkening & $0.2762_{-0.0040}^{+0.0037}$ & $0.2762_{-0.0040}^{+0.0037}$ & $0.2762_{-0.0041}^{+0.0038}$ & $0.2764_{-0.0040}^{+0.0038}$ \\
\hline$u_{1 R}$ & Linear Limb-darkening & $0.385 \pm 0.011$ & $0.385 \pm 0.011$ & $0.385 \pm 0.011$ & $0.385 \pm 0.011$ \\
\hline$u_{2 R}$ & Quadratic Limb-darkening & $0.2739_{-0.0060}^{+0.0054}$ & $0.2740_{-0.0059}^{+0.0054}$ & $0.2740_{-0.0059}^{+0.0055}$ & $0.2742_{-0.0058}^{+0.0055}$ \\
\hline$u_{1 \text { Sloani }}$ & Linear Limb-darkening & $0.3205_{-0.0090}^{+0.0093}$ & $0.3202_{-0.0089}^{+0.0093}$ & $0.3203 \pm 0.0092$ & $0.3199 \pm 0.0091$ \\
\hline$u_{2 S l o a n i}$ & Quadratic Limb-darkening & $0.2761_{-0.0044}^{+0.0040}$ & $0.2762_{-0.0044}^{+0.0040}$ & $0.2761_{-0.0045}^{+0.0040}$ & $0.2763_{-0.0043}^{+0.0040}$ \\
\hline$u_{1 V}$ & Linear Limb-darkening & $0.487_{-0.013}^{+0.014}$ & $0.487 \pm 0.014$ & $0.486 \pm 0.014$ & $0.486 \pm 0.014$ \\
\hline$u_{2 V}$ & Quadratic Limb-darkening & $0.2440_{-0.0089}^{+0.0083}$ & $0.2442_{-0.0087}^{+0.0083}$ & $0.2443_{-0.0087}^{+0.0084}$ & $0.2446_{-0.0087}^{+0.0083}$ \\
\hline \multicolumn{6}{|l|}{ Secondary } \\
\hline \multicolumn{6}{|l|}{ Eclipse } \\
\hline$T_{S}$ & Time of eclipse $\left(\mathrm{BJD}_{\mathrm{TDB}}\right)$ & $2457794.278_{-0.019}^{+0.016}$ & $2457792.90798_{-0.00072}^{+0.00073}$ & $2457794.278_{-0.019}^{+0.016}$ & $2457792.90796_{-0.00070}^{+0.00071}$ \\
\hline$b_{S}$ & Impact parameter & $0.30_{-0.19}^{+0.15}$ & $\cdots$ & $0.32_{-0.20}^{+0.15}$ & $\cdots$ \\
\hline$T_{S, F W H M}$ & FWHM duration (days) & $0.0851_{-0.0050}^{+0.0042}$ & $\cdots$ & $0.0850_{-0.0053}^{+0.0045}$ & $\cdots$ \\
\hline$\tau_{S}$ & $\begin{array}{l}\text { Ingress/egress dura- } \\
\text { tion (days) }\end{array}$ & $0.0114_{-0.0012}^{+0.0020}$ & $\cdots$ & $0.0116_{-0.0013}^{+0.0022}$ & $\cdots$ \\
\hline$T_{S, 14}$ & Total duration (days) & $0.0969_{-0.0059}^{+0.0052}$ & $\cdots$ & $0.0971_{-0.0064}^{+0.0055}$ & $\cdots$ \\
\hline$P_{S}$ & $\begin{array}{l}\text { A priori non-grazing } \\
\text { eclipse probability }\end{array}$ & $0.1801_{-0.0073}^{+0.013}$ & $\cdots$ & $0.1810_{-0.0080}^{+0.014}$ & $\cdots$ \\
\hline$P_{S, G}$ & A priori eclipse probability & $0.2292_{-0.0097}^{+0.018}$ & $\cdots$ & $0.230_{-0.011}^{+0.019}$ & $\cdots$ \\
\hline
\end{tabular}

Note. The $T_{C}$ values are the times of inferior conjunction derived from the individual follow-up light curves.

280 , or $1100 M_{J}$ if its semimajor axis is $0.5,1,5$, or 10 au. The last of these, and any farther out more massive object, is easily ruled out as the cause of the observed RV trend by the lack of a second set of lines in the TRES spectra and the absence of a second source in the AO images (Section 2.4). Besides KELT$22 \mathrm{~B}$ at a projected distance of $6 . " 1$, there is no evidence for another stellar companion close to KELT-22A (Section 2.4). Additional RV measurements over a longer observational baseline are required to either confirm and refine the linear RV trend, or alleviate the need to invoke any RV trend beyond that caused by KELT-22Ab. If this $-2.06_{-0.91}^{+0.93} \mathrm{~m} \mathrm{~s}^{-1}$ day $^{-1}$ trend is real, it may be indicative of an additional sub-stellar body within a few astronomical units, or a somewhat farther-out late M-dwarf, gravitationally bound to KELT-22A.

\subsection{Light-curve Frequency Analysis}

During the initial candidate selection process, it was apparent that the KELT light curve of this system displayed sinusoidal signals with periods of around eight days. This motivated a more targeted analysis of periodic signals (in addition to the planetary transit). A Lomb-Scargle (LS; Press et al. 1992; Zechmeister \& Kürster 2009) analysis was performed to search for periodic sinusoidal signals in the survey light curve, as implemented in the VARTOOLS light curve analysis package (Hartman 2012). The computed periodogram is shown in Figure 11 (upper panel). We do not detect a single dominant frequency, but rather a forest of peaks is evident with periods of 7-9 days. The top few peaks in this region are all of similar significance, and are well above the noise. The period, formal false-alarm probability (FAP), and signal-to-noise ratio $(\mathrm{S} / \mathrm{N})$ associated with these peaks are listed in Table 5. The periodogram peaks near one day and integer fractions of one day are aliases caused by the diurnal observing cycle of KELT.

The periodogram shown in Figure 11 is calculated from the entire KELT light curve, and the top peak is $P_{0}=8.2763 d$. The entire light curve phased to this peak is shown in the bottom left panel of Figure 11. Significant peaks of slightly different periods are found when different segments of the light curve are analyzed in this fashion. Regardless of the section of the KELT light curve analyzed, all of the significant peaks lie within the forest seen between 7 and 9 days. 

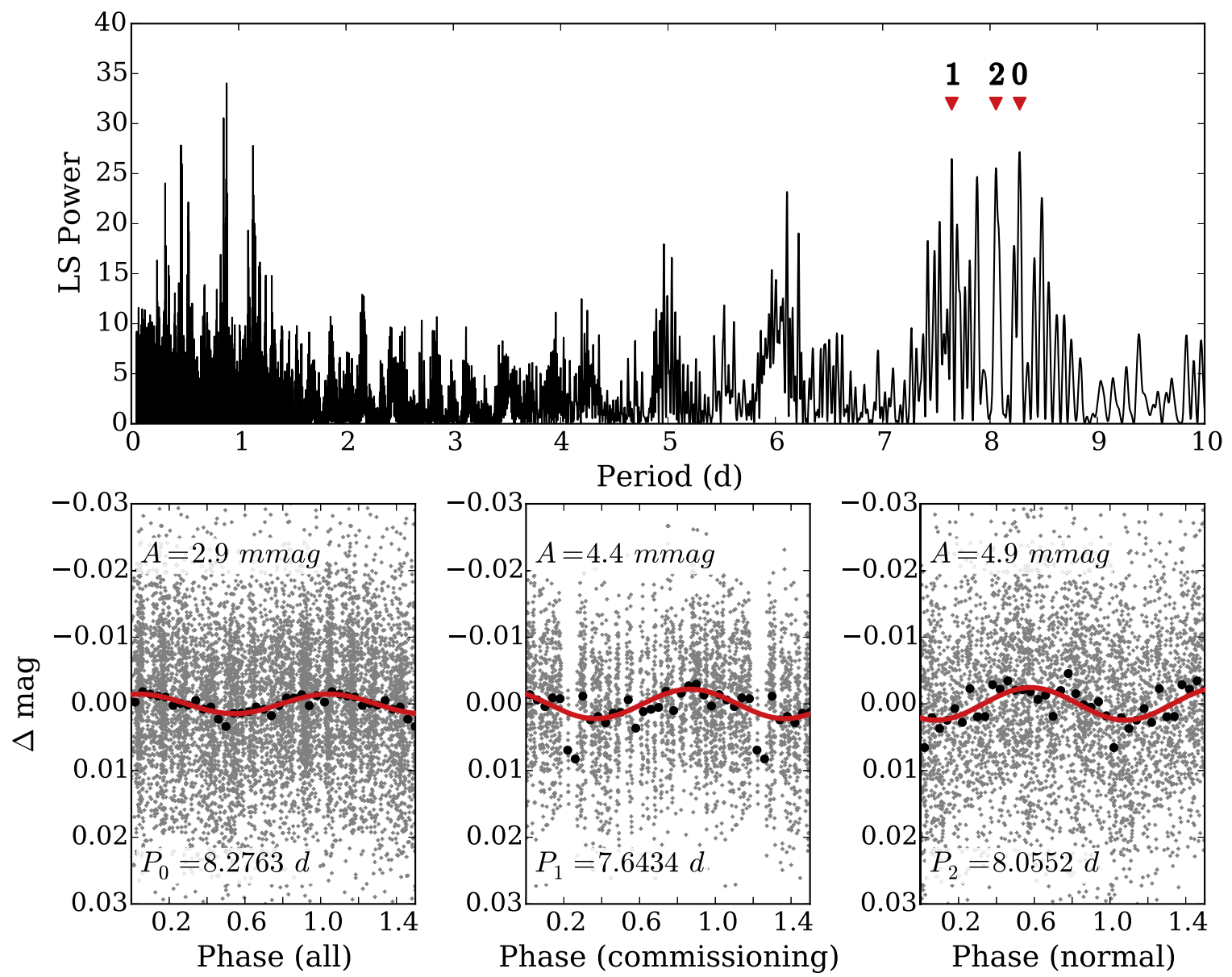

Figure 11. Top panel shows the Lomb-Scargle periodogram computed from the survey data for KELT-22. There is a forest of peaks between 7 and 9 days. The lower row shows the photometry phased to the periods associated with the strongest LS peaks, with all observations on the left, only commissioning data in the middle, and all of the normal science operation data on the right. The gray points show each observation, the black points show the data binned with 25 bins in phase, and the red curve is a single-component sinusoidal fit to the binned data. The peak-to-trough amplitude of the sinusoidal fit is given in each of these panels.

Table 5

Periodic Signals Detected in Photometry

\begin{tabular}{lccc}
\hline \hline & $\begin{array}{c}\text { Period } \\
(\text { d) }\end{array}$ & $\log ($ FAP $)$ & SNR \\
\hline$P_{0}$ & 8.2763 & -6.94 & 21.2 \\
$P_{1}$ & 7.6434 & -6.64 & 20.7 \\
$P_{2}$ & 8.0552 & -6.24 & 19.9 \\
$P_{3}$ & 7.8804 & -5.87 & 19.2 \\
\hline
\end{tabular}

Note. Table of periods detected in the light curve of KELT-22 that are possibly associated with stellar rotation.

The commissioning data span 95 days, with dense time coverage and a total of 2294 observations. The most significant peak from this section of the light curve is $P_{1}=7.6434 d$. The commissioning data are phased to this period in the bottom middle panel of Figure 11. Normal science operation of KELTSouth begins 612 days after the commissioning phase is completed, and spans 1072 days with 2918 observations. An LS periodogram is again computed for only the data taken during normal science operation. The top LS peak for this section of the light curve is $P_{2}=8.0552$ days. The bottom right panel of Figure 11 shows the normal science data phased to this period.

It is possible that these signals are associated with rotation of KELT-22A. Starspots on the stellar surface can rotate into and out of view, causing variation in the observed brightness at the rotation period of the star (e.g., Evans 1971; Cargile et al. 2014). Starspots in Sun-like stars are not permanent features, but rather have finite lifetimes that can depend on a number of conditions (e.g., Bradshaw \& Hartigan 2014; Giles et al. 2017). From our Sun, we can measure the solar rotation period from sunspots, and find that it is latitude-dependentthat is, the Sun is differentially rotating. A frequency analysis of a differentially rotating star with transient star spots may be consistent with the periodogram features that we see in KELT-22A (Reinhold et al. 2013). The NUV excess in the SED of KELT-22A points to enhanced chromospheric activity, which could be consistent with the magnetic activity that star spots are correlated with. These findings are suggestive, but are insufficient to claim this as the definitive cause of the forest of peaks in the LS periodogram. A more detailed study is required to further address this hypothesis. Regardless of the physical interpretation, because the light from KELT-22A and its companion are fully blended in KELT, we have no way to know from which source these signals arise with the available data (although we note that KELT-22A contributes about twice as much flux to the KELT light curve relative to KELT-22B).

Additionally, our measured $v \sin I_{\star}$ and $R_{\star}$ values imply that the stellar rotation period of KELT-22A should be $\sim 7.5$ days 

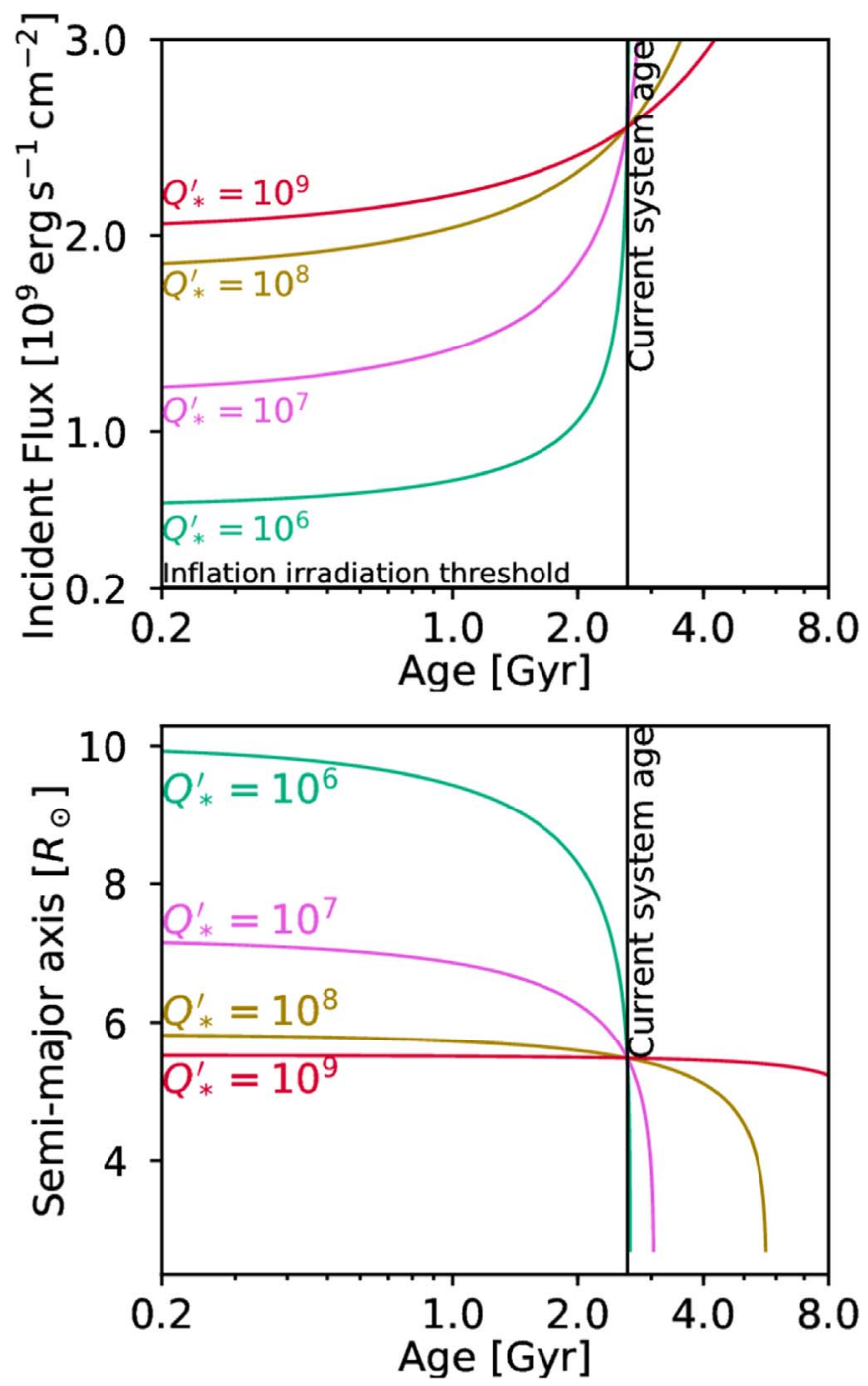

Figure 12. Top: evolution of the amount of flux incident on KELT-22Ab, predicted for different values of $Q_{\star}^{\prime}$. According to these models, for KELT$22 \mathrm{Ab}$ the incident flux has always been above the inflation irradiation threshold of $2 \times 10^{8} \mathrm{erg} \mathrm{s}^{-1} \mathrm{~cm}^{-2}$ identified by Demory \& Seager (2011). Bottom: change in semimajor axis for KELT-22Ab shown for a range of values for $Q_{\star}^{\prime}$.

(assuming $\sin I_{\star} \sim 1$ ), which is consistent with the forest of peaks in the periodogram. This suggests that this signal in the light curve is consistent with being due to the rotation of KELT-22A, but again we cannot confirm whether this is the case with our current data.

\subsection{Tidal Evolution and Irradiation History}

Using the parameters derived from our global fit as boundary conditions, we simulate the past and future evolution of the orbit of KELT-22Ab using the POET code (Penev et al. 2014), under the assumptions of a circular orbit, no other perturbing body, and a constant phase lag (constant tidal quality factor). This simulation examines the changes in the semimajor axis that arise from tidal forces acting between star and planet, and also the changes in incident flux (both due to the diminishing semimajor axis and the increasing luminosity). The strength of these tidal forces is often parameterized by the tidal dissipation parameter $\left(Q_{\star}^{\prime}\right)$, which is defined as the tidal quality factor divided by the love number $\left(Q_{\star}^{\prime} \equiv Q_{\star} / k_{2}\right)$. We test values from $\log Q_{\star}^{\prime}=6-9$. Higher values of $Q_{\star}^{\prime}$ are less dissipative, while lower values allow tidal forces to dissipate energy quickly, forcing more rapid changes in the system.

An irradiation threshold of $\sim 2 \times 10^{8} \mathrm{erg} \mathrm{s}^{-1} \mathrm{~cm}^{-2}$ has been found, above which giant planets tend to become inflated (Demory \& Seager 2011). KELT-22Ab is above this threshold at present (by about a factor of ten). Our model shows that regardless of the choice of $Q_{\star}^{\prime}$, KELT-22Ab has been above this threshold throughout its entire evolutionary history (see the upper panel of Figure 12). The planet is inflated, as evidenced by its large radius $\left(R_{P}=1.285_{-0.071}^{+0.12} R_{J}\right)$. This observation is consistent with a history of such high irradiation.

The orbital evolution of KELT-22Ab predicted by our model depends strongly on the value of $Q_{\star}^{\prime}$. For lower values of $Q_{\star}^{\prime}$ $\left(\log Q_{\star}^{\prime}\right.$ of 6 and 7$)$ that we tested, the planet is predicted to spiral into the star within the next few hundred Myr, while larger values of $Q_{\star}^{\prime}$ will allow the planet to survive for longer. The orbital evolution calculations presented in Figure 12 (lower panel) show that for a value of $\log Q_{\star}^{\prime}=6$, which may be appropriate for many hot Jupiter systems (Penev et al. 2018), KELT-22Ab is moving very quickly through its semimajor axis. This prediction means that the planet spends only a small fraction of the stellar main-sequence lifetime in the orbit that we observe at present. Systems like KELT-22A should then be intrinsically rare if these tidal evolution models are reliable, as the orbits are very quickly evolving toward engulfment (despite the strong observational biases of transit and RV exoplanet surveys toward hot Jupiters). Collier Cameron \& Jardine (2018), and Penev et al. (2012) years before, used this reasoning to argue against such low values of $Q_{\star}^{\prime}$ being typical. However, there is still much uncertainty in this field and stronger observational constraints are required.

Penev et al. (2018) provided a formula to calculate $Q_{\star}^{\prime}$ with knowledge of the orbital period and the stellar rotation period. If the sinusoidal signals discussed in Section 6.2 are in fact caused by the rotation of KELT-22A (which is generally in agreement with the spectroscopically derived $v \sin I_{\star}$ ), this gives us an estimate of the stellar rotational period. To proceed, we assume that the most significant peak in the periodogram $\left(P_{\text {spin }}=8.2763\right.$ days $)$ is the stellar rotation period. We use the orbital period from the global fit $\left(P_{\text {orb }}=1.3867\right.$ days $)$. We then find that $\log Q_{\star}^{\prime}=6.246$. This calculation is not extremely sensitive to the stellar spin period. If we instead use the shortest significant LS peak as the stellar rotation period, the result is essentially the same, $\log Q_{\star}^{\prime}=6.224$. This value of $\log$ $Q_{\star}^{\prime} \sim 6.2$ for KELT-22A is generally consistent with values for $\log Q_{\star}^{\prime}$ calculated for dozens of hot Jupiter systems in Penev et al. (2018), and suggests a strong coupling between the planetary orbit and stellar rotation, where tidal forces are causing the stellar rotation rate to increase, at the expense of a decrease in the semimajor axis. Given the stated assumptions, this orbital evolution model predicts that KELT-22Ab is moving through this semimajor axis quickly and will be consumed by its star within the next few hundred Myr.

Considering hot Jupiters in close-in orbits around Sun-like stars (having convective envelopes and radiative cores), there is a point where tidal dissipation forces transition from weakly to strongly nonlinear. This transition is heavily dependent on the semimajor axis, planetary mass, and the stellar age and internal structure. With a small semimajor axis $\left(a / R_{\star}=4.97\right)$ and large planetary mass $\left(3.47 M_{J}\right)$, the KELT-22A system is likely near this boundary (Barker \& Ogilvie 2010). The theoretical calculations of Essick \& Weinberg (2016) assume weakly 

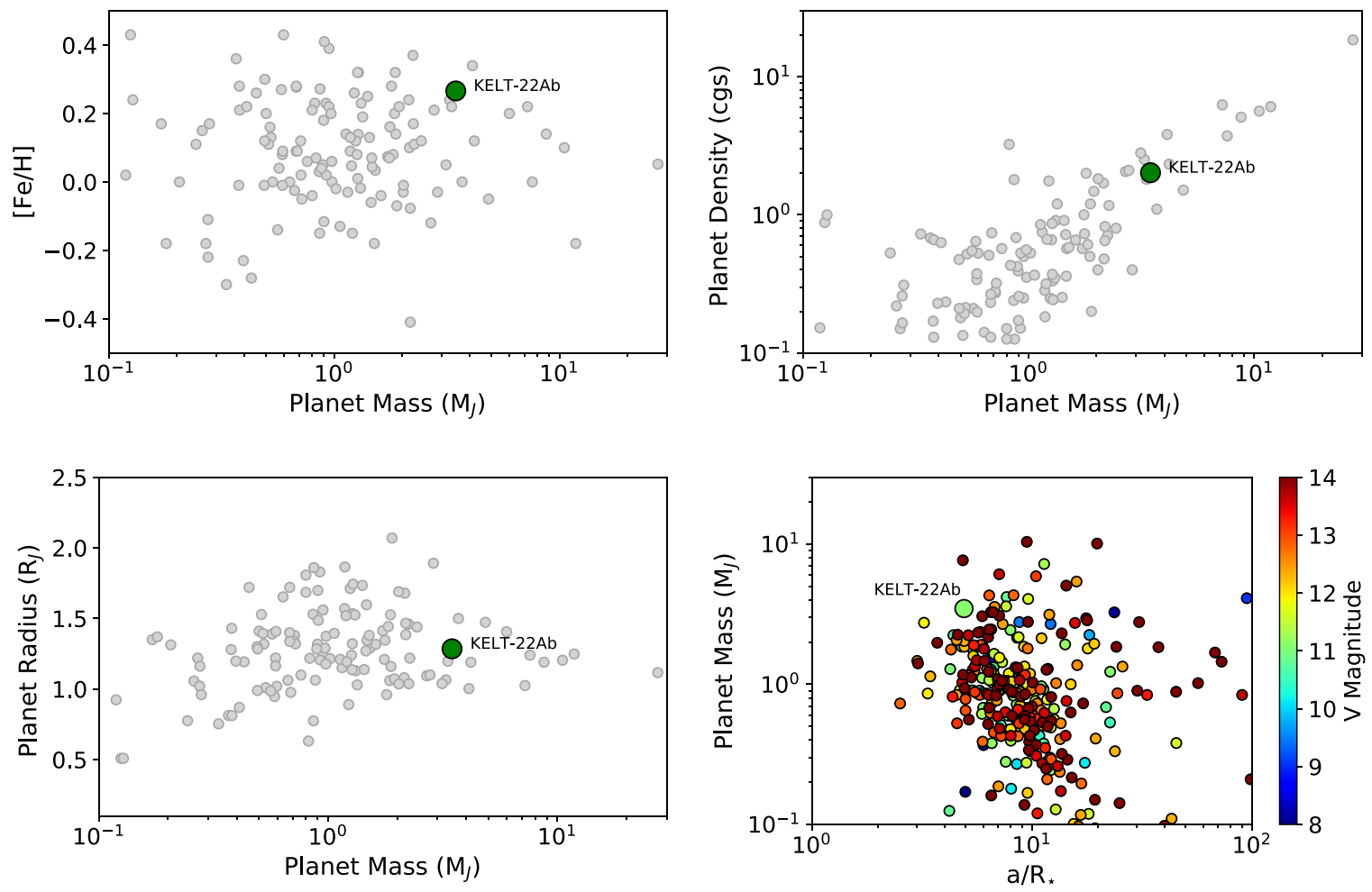

Figure 13. Plots showing stellar metallicity, planetary density, and planetary radius vs. planetary mass for known transiting exoplanets with $K \lesssim 10$ (top row and bottom left panel). In this brightness regime, KELT-22Ab is among the most massive and has a somewhat high bulk density, with a metal-rich host.The bottom right panel shows planet mass vs. semimajor axis divided by stellar radius for all known transiting planets having a host star with $T_{\text {eff }}<6250 \mathrm{~K}$ (i.e., being below the Kraft break and likely having a convective envelope), with the symbol color scaled with the host star $V$-band magnitude. Bright stars that host transiting massive planets at small $a / R_{\star}$ are well suited for studying the effects of tidal interactions between stars and planet. Data are from TEPCat (Southworth 2011), accessed on 2018 February 18.

nonlinear tidal dissipation, and predict values of $\log Q_{\star}^{\prime} \sim 6.1$ and an orbital decay time of $\tau \sim 200 \mathrm{Myr}$ for the KELT-22A system. This is generally in agreement with values calculated using the methods of Penev et al. (2018) in the previous paragraph, which also assume weakly nonlinear tidal dissipation. If instead the dynamical tide is in the strongly nonlinear regime, Barker \& Ogilvie (2010) predicted a value of $\log$ $Q_{\star}^{\prime} \sim 5$ 5.6. Strongly nonlinear tides would result in relatively rapid in-spiral of the planet.

The inferred $\sim 8$ day spin period of KELT-22A may be relevant to this discussion, as it is significantly shorter than expected given the Skumanich law for Sun-like stars. The rapid stellar rotation may be explained by youth, or this may be a natural consequence of tidal spin-up. These two scenarios are not mutually exclusive, although if the tidal forces are on the high side of the models considered here, then the orbit is shrinking very rapidly, so the present configuration likely exists for only a very small fraction of the stellar main-sequence lifetime, perhaps suggesting that youth is the dominant reason for the rapid rotation.

The tidal dissipation models considered here predict rapid orbital evolution toward smaller star-planet separation. However, the details are largely uncertain. With future transit observations, it may be possible to directly measure the decay of the orbit using methods similar to those applied to WASP-12 (Hebb et al. 2009; Maciejewski et al. 2016; Patra et al. 2017). Equation (20) of Goldreich \& Soter (1966) provides a formula to calculate the rate of change of the semimajor axis from tidal dissipation: $\frac{d a}{d t}=\dot{a}=-\frac{9}{2} \sqrt{\frac{G}{a M_{\star}}}\left(\frac{R_{\star}}{a}\right)^{5}\left(\frac{M_{P}}{Q_{\star}^{\prime}}\right)$, where $G$ is the gravitational constant, and the other symbols are as previously defined. This expression assumes that the mass of the planet is negligible compared to the mass of the star. We use this equation, along with Kepler's third law $\left(\frac{\dot{P}}{P}=1.5 \frac{\dot{a}}{a}\right)$ and the system parameters from the global fit to calculate the derivative of the orbital period. We assume a circular orbit and a constant period derivative $\left(P(t)=P_{0}+\dot{P} t\right)$. We then use the calculated $\dot{P}$ to find the drift of the time of transit center with the equation $\Delta T_{c}=\frac{1}{2} t^{2} \frac{\dot{P}}{P}$. With $Q_{\star}^{\prime}=10^{6}$, we find $\Delta T_{c} \sim 19 \mathrm{~s}$ for $t=$ $10 \mathrm{yr}$, or $\Delta T_{c} \sim 75 \mathrm{~s}$ for $t=20 \mathrm{yr}$. These drifts are inversely proportional to $Q_{\star}^{\prime}$. Continued observations of KELT-22A may eventually be used to directly measure $\Delta T_{c}$. In this context the KELT-22A system is an excellent candidate to directly test theories that describe the tidal interactions between hot Jupiters with Sun-like hosts.

\subsection{Predictions for Measuring Spin-Orbit Misalignment}

With $v \sin I_{\star}=7.9 \pm 0.5 \mathrm{~km} \mathrm{~s}^{-1}$, KELT-22A is rotating relatively rapidly for an early $\mathrm{G}$ star. It is thus a good target for measurement of the angle between the planetary orbit and the stellar spin axis via the Rossiter-McLaughlin effect or Doppler tomography. Based upon the system parameters and the formulae of Gaudi \& Winn (2007), we estimate that KELT$22 \mathrm{Ab}$ should have a Rossiter-McLaughlin semi-amplitude of $\sim 120 \mathrm{~m} \mathrm{~s}^{-1}$. This large value is thanks to the relatively large $v \sin I_{\star}$ and transit depth. This should be easily measurable for a $V=11.1$ star like KELT-22A, even with the relatively short $2.4 \mathrm{hr}$ transit duration. This will be interesting in light of the bound stellar companion, and also the possibility of an 
additional, possibly sub-stellar, companion. Such observations serve to test the proposed mechanisms that could generate misaligned hot Jupiters that rely on the presence of an additional object in the system (e.g., Fabrycky \& Tremaine 2007; Naoz et al. 2013). This (mis-)alignment may also be relevant in the tidal interactions between star and planet.

\subsection{Comparative Planetology}

Many factors influence the formation of planetary systems. Among these are stellar multiplicity and metallicity. With the bound stellar companion and a metal-rich composition, KELT$22 \mathrm{~A}$ further fills in this parameter space, with a host star bright enough for detailed characterization studies (see Figure 13). The metallicity, density, and radius of known transiting exoplanets (including KELT-22Ab) are plotted against mass in Figure 13.

If our models of the tidal forces between star and planet are even approximately correct, then KELT-22Ab is rapidly moving through this stage in its orbital evolution, and provides a valuable opportunity to constrain the behavior of tides in such systems. In particular, the small semimajor axis, large planetary mass, brightness of the host star, and structure of the host star (i.e., likely having a radiative core and a convective envelope) make this a prime system for studying tidal interactions between star and planet (see the bottom right panel in Figure 13). The hint of a third (possibly non-stellar) body in this system (as implied by the linear RV slope), the likely young age, and the very peculiar space motion through the Galaxy, motivate further study.

\section{Conclusion and Future Work}

We present the discovery of KELT-22Ab, a hot Jupiter transiting the bright $V=11.1, K=10.0$ Sun-like G2 star TYC 7518-468-1. The planet is massive $\left(M_{P}=3.47_{-0.14}^{+0.15} M_{J}\right)$, large $\left(R_{P}=1.285_{-0.071}^{+0.12} R_{J}\right)$, and on a short 1.39 day orbital period. KELT-22A is metal-rich $([\mathrm{m} / \mathrm{H}]=0.26)$ and has an unusually high space velocity, with excursions up to $\sim 280 \mathrm{pc}$ out of the galactic plane. KELT-22A rotates relatively rapidly for a mainsequence G2 star, with $v \sin I_{\star}=7.9 \pm 0.5 \mathrm{~km} \mathrm{~s}^{-1}$, and with photometric hints of a rotational period of $\sim 8$ days. Models that simulate the tidal interactions between star and planet predict that tidal forces are spinning up the star, with the consequence that the planet is losing orbital angular momentum, perhaps causing it to spiral into the star within the next few hundred Myr. Continued photometric observations of the transit can be used to measure or constrain the system's orbital evolution. The brightness and relatively rapid rotation of KELT-22A and large transit depth make this an excellent candidate for measuring the spin-orbit alignment through either the Rossiter-McLaughlin effect or Doppler tomography. A linear trend in RV measurements hints at the possibility of a third body in the system (in addition to the widely separated binary companion). Future RV monitoring of the KELT-22A system is therefore warranted to either confirm or rule out this trend.

During the completion of this paper, we became aware of an independent discovery of this planetary system by the SuperWASP survey (WASP-173b; Hellier et al. 2019). Since the data we present in this paper were collected independently and the analysis was performed before the announcement of WASP-173b, we have chosen to discuss our findings as an independent discovery of this planet, and we refer to it here as KELT-22Ab. However, we acknowledge the prior announcement of it as WASP-173b.

This project makes use of data from the KELT survey, including support from The Ohio State University, Vanderbilt University, and Lehigh University, along with the KELT follow-up collaboration. J.L.-B. acknowledges support from FAPESP (grant 2017/23731-1). Work performed by J.E.R. was supported by the Harvard Future Faculty Leaders Postdoctoral fellowship. D.W.L. acknowledges partial support under NASA Cooperative Agreement NNX13AB58A with the Smithsonian Astrophysical Observatory. D.J.S. and B.S.G. were partially supported by NSF CAREER grant AST1056524. D.J.J. gratefully acknowledges support from National Science Foundation award NSF-1440254. Work by S.V.Jr. is supported by the National Science Foundation Graduate Research Fellowship under grant No. DGE-1343012 and the David G. Price Fellowship in Astronomical Instrumentation. Work by G.Z. is provided by NASA through Hubble Fellowship grant HST-HF2-51402.001-A awarded by the Space Telescope Science Institute, which is operated by the Association of Universities for Research in Astronomy, Inc., for NASA, under contract NAS 5-26555. This work has made use of NASA's Astrophysics Data System, the Extrasolar Planet Encyclopedia, the NASA Exoplanet Archive, the SIMBAD database operated at CDS, Strasbourg, France, and the VizieR catalog access tool, CDS, Strasbourg, France. We also used data products from the Widefield Infrared Survey Explorer, which is a joint project of the University of California, Los Angeles; the Jet Propulsion Laboratory/California Institute of Technology, which is funded by the National Aeronautics and Space Administration; the Two Micron All Sky Survey, which is a joint project of the University of Massachusetts and the Infrared Processing and Analysis Center/California Institute of Technology, funded by the National Aeronautics and Space Administration and the National Science Foundation; and the European Space Agency (ESA) mission Gaia (http://www. cosmos.esa.int/gaia), processed by the Gaia Data Processing and Analysis Consortium (DPAC, http://www.cosmos.esa. int/web/gaia/dpac/consortium). Funding for the DPAC has been provided by national institutions, in particular the institutions participating in the Gaia Multilateral Agreement. MINERVA is a collaboration among the Harvard-Smithsonian Center for Astrophysics, The Pennsylvania State University, the University of Montana, and the University of New South Wales. MINERVA is made possible by generous contributions from its collaborating institutions and Mt. Cuba Astronomical Foundation, The David \& Lucile Packard Foundation, National Aeronautics and Space Administration (EPSCOR grant NNX13AM97A), The Australian Research Council (LIEF grant LE140100050), and the National Science Foundation (grants 1516242 and 1608203). The Center for Exoplanets and Habitable Worlds is supported by the Pennsylvania State University, the Eberly College of Science, and the Pennsylvania Space Grant Consortium. Any opinions, findings, and conclusions or recommendations expressed are those of the author and do not necessarily reflect the views of the National Science Foundation.

The authors thank Andrew Collier Cameron and Nevin N. Weinberg for helpful discussion regarding the role of tidal 
forces in the KELT-22A system, and the anonymous referee for comments that improved this manuscript.

Facilities: KELT, FLWO (TRES), Keck (NIRC2), ANU:2.3 m (WiFeS), LCO.

Software: Python, IDL, IRAF, TAPIR (Jensen 2013), AstroImageJ (Collins \& Kielkopf 2013), EXOFAST (Eastman et al. 2013), POET (Penev et al. 2014), galpy (Bovy 2015), VARTOOLS (Hartman 2012).

\section{ORCID iDs}

Jonathan Labadie-Bartz (iD https://orcid.org/0000-00022919-6786

Joseph E. Rodriguez (iD https://orcid.org/0000-00018812-0565

Keivan G. Stassun (iD https://orcid.org/0000-0002-3481-9052 Kaloyan Penev (1D) https://orcid.org/0000-0003-4464-1371 Marshall C. Johnson (iD https://orcid.org/0000-00025099-8185

B. Scott Gaudi (iD https://orcid.org/0000-0003-0395-9869 Knicole D. Colón (iD https://orcid.org/0000-0001-8020-7121 Allyson Bieryla (iD https://orcid.org/0000-0001-6637-5401 David W. Latham (ib https://orcid.org/0000-0001-9911-7388 Joshua Pepper (10) https://orcid.org/0000-0002-3827-8417 Karen A. Collins (1D https://orcid.org/0000-0001-6588-9574 Robert J. Siverd (iD https://orcid.org/0000-0001-5016-3359 Xinyu Yao (i) https://orcid.org/0000-0003-4554-5592 Thiam-Guan Tan (iD https://orcid.org/0000-0001-5603-6895 George Zhou (iD https://orcid.org/0000-0002-4891-3517 Jason D. Eastman (i) https://orcid.org/0000-0003-3773-5142 Michael D. Albrow (10) https://orcid.org/0000-0003-3316-4012 Daniel Bayliss (1D https://orcid.org/0000-0001-6023-1335 Thomas G. Beatty (i) https://orcid.org/0000-0002-9539-4203 Valerio Bozza (1D https://orcid.org/0000-0003-4590-0136 Michael L. Calkins (10) https://orcid.org/0000-0002-2830-5661 Dax Feliz (iD https://orcid.org/0000-0002-2457-7889

Benjamin J. Fulton (iD https://orcid.org/0000-0003-3504-5316 Eric L. N. Jensen (iD https://orcid.org/0000-0002-4625-7333 Michael B. Lund (ib https://orcid.org/0000-0003-2527-1598 Mark Manner (iD https://orcid.org/0000-0002-9411-7271 Kim K. McLeod (1) https://orcid.org/0000-0001-9504-1486 Matthew T. Penny (iD https://orcid.org/0000-0001-7506-5640 Phillip A. Reed (i) https://orcid.org/0000-0002-5005-1215 Daniel J. Stevens (iD https://orcid.org/0000-0002-5951-8328 Steven Villanueva, Jr. (i) https://orcid.org/0000-00016213-8804

Robert A. Wittenmyer (i) https://orcid.org/0000-00019957-9304

J. T. Wright (iD https://orcid.org/0000-0001-6160-5888

\section{References}

Alonso, R., Brown, T. M., Torres, G., et al. 2004, ApJL, 613, L153 Alsubai, K. A., Parley, N. R., Bramich, D. M., et al. 2013, AcA, 63, 465 Astraatmadja, T. L., \& Bailer-Jones, C. A. L. 2016, ApJ, 833, 119 Baglin, A., Auvergne, M., Boisnard, L., et al. 2006, 36th COSPAR Assembly (Göttingen: Copernicus)

Bakos, G., Noyes, R. W., Kovács, G., et al. 2004, PASP, 116, 266

Barker, A. J., \& Ogilvie, G. I. 2010, MNRAS, 404, 1849

Bayliss, D., Zhou, G., Penev, K., et al. 2013, AJ, 146, 113

Bensby, T., Feltzing, S., \& Lundström, I. 2003, A\&A, 410, 527

Berta, Z. K., Irwin, J., Charbonneau, D., Burke, C. J., \& Falco, E. E. 2012, AJ, 144,145

Bianchi, L., Herald, J., Efremova, B., et al. 2011, Ap\&SS, 335, 161

Borucki, W. J., Koch, D., Basri, G., et al. 2010, Sci, 327, 977
Bovy, J. 2017, MNRAS, 470, 1360

Bovy, J. 2015, ApJS, 216, 29

Bowler, B. P. 2016, PASP, 128, 102001

Bradshaw, S. J., \& Hartigan, P. 2014, ApJ, 795, 79

Buchhave, L. A., Bakos, G. Á, Hartman, J. D., et al. 2010, ApJ, 720, 1118 Buchhave, L. A., Latham, D. W., Johansen, A., et al. 2012, Natur, 486, 375

Cardelli, J. A., Clayton, G. C., \& Mathis, J. S. 1989, ApJ, 345, 245

Cargile, P. A., James, D. J., Pepper, J., et al. 2014, ApJ, 782, 29

Choi, J., Dotter, A., Conroy, C., et al. 2016, ApJ, 823, 102

Ciardi, D. R., Beichman, C. A., Horch, E. P., \& Howell, S. B. 2015, ApJ, 805, 16 Ciardi, D. R., Crossfield, I. J. M., Feinstein, A. D., et al. 2018, AJ, 155, 10

Ciardi, D. R., von Braun, K., Bryden, G., et al. 2011, AJ, 141, 108

Claret, A., \& Bloemen, S. 2011, A\&A, 529, A75

Collier Cameron, A., \& Jardine, M. 2018, MNRAS, 476, 2542

Collins, K., \& Kielkopf, J. 2013, AstroImageJ: ImageJ for Astronomy, Astrophysics Source Code Library, ascl:1309.001

Collins, K. A., Collins, K. I., Pepper, J., et al. 2018, AJ, 156, 234

Collins, K. A., Kielkopf, J. F., Stassun, K. G., \& Hessman, F. V. 2017, AJ, 153,77

Coşkunoğlu, B., Ak, S., Bilir, S., et al. 2011, MNRAS, 412, 1237

Cutri, R. M., Wright, E. L., Conrow, T., et al. 2013, yCat, 2328

Cutri, R. M., Skrutskie, M. F., van Dyk, S., et al. 2003, yCat, 2246, 0

Dawson, R. I., \& Johnson, J. A. 2018, MNRAS, 56, 175

Demory, B.-O., \& Seager, S. 2011, ApJS, 197, 12

Desort, M., Lagrange, A.-M., Galland, F., Udry, S., \& Mayor, M. 2007, A\&A, 473, 983

Dopita, M., Hart, J., McGregor, P., et al. 2007, Ap\&SS, 310, 255

Dotter, A. 2016, ApJS, 222, 8

Eastman, J., Gaudi, B. S., \& Agol, E. 2013, PASP, 125, 83

Eastman, J. D., Beatty, T. G., Siverd, R. J., et al. 2016, AJ, 151, 45

Essick, R., \& Weinberg, N. N. 2016, ApJ, 816, 18

Evans, D. S. 1971, MNRAS, 154, 329

Fabricius, C., Høg, E., Makarov, V. V., et al. 2002, A\&A, 384, 180

Fabrycky, D., \& Tremaine, S. 2007, ApJ, 669, 1298

Fürész, G., Szentgyorgyi, A. H., \& Meibom, S. 2008, in Proc ESO Conf. Precision Spectroscopy in Astrophysics, ed. N. C. Santos et al. (Garching: ESO), 287

Furlan, E., Ciardi, D. R., Everett, M. E., et al. 2017, AJ, 153, 71

Gaia Collaboration, Brown, A. G. A., Vallenari, A., et al. 2018, A\&A, 616, A1 Gaudi, B. S., \& Winn, J. N. 2007, ApJ, 655, 550

Giles, H. A. C., Collier Cameron, A., \& Haywood, R. D. 2017, MNRAS, 472, 1618

Gillon, M., Triaud, A. H. M. J., Demory, B.-O., et al. 2017, Natur, 542, 456

Goldreich, P., \& Soter, S. 1966, Icar, 5, 375

Hartman, J. 2012, VARTOOLS: Light Curve Analysis Program, Astrophysics Source Code Library, ascl:1208.016

Hebb, L., Collier-Cameron, A., Loeillet, B., et al. 2009, ApJ, 693, 1920

Hellier, C., Anderson, D. R., Bouchy, F., et al. 2019, MNRAS, 482, 1379

Henden, A. A., Levine, S., Terrell, D., \& Welch, D. L. 2015, AAS Meeting Abstracts, 225, 336.16

Høg, E., Fabricius, C., Makarov, V. V., et al. 2000, A\&A, 355, L27

Howell, S. B., Sobeck, C., Haas, M., et al. 2014, PASP, 126, 398

Jensen, E. 2013, Tapir: A web interface for transit/eclipse observability, Astrophysics Source Code Library, ascl:1306.007

Kuhn, R. B., Rodriguez, J. E., Collins, K. A., et al. 2016, MNRAS, 459, 4281

Kurucz, R. L. 1992, in IAU Symp. 149, The Stellar Populations of Galaxies, ed. B. Barbuy \& A. Renzini (Dordrecht: Kluwer), 225

Lutz, T. E., \& Kelker, D. H. 1973, PASP, 85, 573

Maciejewski, G., Dimitrov, D., Fernández, M., et al. 2016, A\&A, 588, L6

McCullough, P. R., Stys, J. E., Valenti, J. A., et al. 2005, PASP, 117, 783

Mortier, A., Sousa, S. G., Adibekyan, V. Z., Brandão, I. M., \& Santos, N. C. 2014, A\&A, 572, A95

Naoz, S., Farr, W. M., Lithwick, Y., Rasio, F. A., \& Teyssandier, J. 2013, MNRAS, 431, 2155

Ngo, H., Knutson, H. A., Hinkley, S., et al. 2016, ApJ, 827, 8

Nutzman, P., Charbonneau, D., Irwin, J., et al. 2009, AAS Meeting Abstracts, $41,8.02$

O’Donovan, F. T., Charbonneau, D., Torres, G., et al. 2006, ApJ, 644, 1237

Patra, K. C., Winn, J. N., Holman, M. J., et al. 2017, AJ, 154, 4

Penev, K., Bouma, L. G., Winn, J. N., \& Hartman, J. D. 2018, AJ, 155, 155

Penev, K., Jackson, B., Spada, F., \& Thom, N. 2012, ApJ, 751, 96

Penev, K., Zhang, M., \& Jackson, B. 2014, PASP, 126, 553

Pepper, J., Kuhn, R. B., Siverd, R., James, D., \& Stassun, K. 2012, PASP, 124,230

Pepper, J., Pogge, R. W., DePoy, D. L., et al. 2007, PASP, 119, 923

Platais, I., Girard, T. M., Kozhurina-Platais, V., et al. 1998, AJ, 116, 2556 
Pollacco, D. L., Skillen, I., Collier Cameron, A., et al. 2006, PASP, 118, 1407 Press, W. H., Teukolsky, S. A., Vetterling, W. T., \& Flannery, B. P. 1992, Numerical Recipes in C. The art of Scientific Computing (2nd ed.; Cambridge: Cambridge Univ. Press)

Raghavan, D., McAlister, H. A., Henry, T. J., et al. 2010, ApJS, 190, 1

Reinhold, T., Reiners, A., \& Basri, G. 2013, A\&A, 560, A4

Röser, S., Schilbach, E., Schwan, H., et al. 2008, A\&A, 488, 401

Schlegel, D. J., Finkbeiner, D. P., \& Davis, M. 1998, ApJ, 500, 525

Seager, S., \& Mallén-Ornelas, G. 2003, ApJ, 585, 1038

Siverd, R. J., Beatty, T. G., Pepper, J., et al. 2012, ApJ, 761, 123
Siverd, R. J., Collins, K. A., Zhou, G., et al. 2018, AJ, 155, 35 Southworth, J. 2011, MNRAS, 417, 2166

Stassun, K. G., \& Torres, G. 2018, ApJ, 862, 61

Szentgyorgyi, A. H., \& Fürész, G. 2007, RMxAC, 28, 129

Torres, G., Andersen, J., \& Giménez, A. 2010, A\&ARv, 18, 67

Torres, G., Konacki, M., Sasselov, D. D., \& Jha, S. 2004, ApJ, 614, 979

Wright, J. T., Marcy, G. W., Howard, A. W., et al. 2012, ApJ, 753, 160

Yi, S., Demarque, P., Kim, Y.-C., et al. 2001, ApJS, 136, 417

Zacharias, N., Finch, C. T., Girard, T. M., et al. 2012, yCat, 1322

Zechmeister, M., \& Kürster, M. 2009, A\&A, 496, 577 